\title{
Multi-Vehicle Coordination in an Unknown Flowfield
}

\author{
Cameron Peterson* and Derek A. Paley ${ }^{\dagger}$ \\ University of Maryland, College Park, MD 20742, USA
}

\begin{abstract}
Cooperative control of autonomous vehicles is a robust approach for many spatiotemporal applications, including area surveillance, target tracking and environmental sampling. Prior research has generated stable control algorithms that promote collaboration of autonomous vehicles. A persistent difficulty in creating control algorithms is accounting for effects due to flowfields such as winds or tides. In this paper we propose decentralized control algorithms that stabilize a group of autonomous vehicles to a circular formation in either an unknown, spatially uniform flowfield or an unknown, spatially uniform flowfield whose direction rotates uniformly in time. The center of the formation can be prescribed or arbitrary. Prescribing the center formation allows us to apply these algorithms to a targettracking problem. We also show how a bounded turning-rate constraint, which is present in many autonomous vehicles, can be incorporated into control algorithms for a known flowfield. The theoretical results are supported by application examples that illustrate multiple vehicles tracking an accelerating target in a circular formation.
\end{abstract}

\section{Nomenclature}

$c_{k} \quad$ Center of circle traversed by particle $k$

$f_{k}(t)$ Flow velocity at position $r_{k}$ and time $t$

$\hat{f}_{k}(t)$ Estimated flow velocity at position $r_{k}$ and time $t$

$i \quad$ Imaginary unit

$K \quad$ Control gain

$N \quad$ Number of particles

$P \quad N \times N$ projector matrix

$P_{k} \quad k$ th row of matrix $P$

$r_{k} \quad$ Position of particle $k$

$\hat{r}_{k} \quad$ Estimated position of particle $k$

$\dot{r}_{k} \quad$ Inertial velocity of particle $k$

$s_{k}(t)$ Inertial speed of particle $k$ at time $t$

$T$ Period of revolution around a circular orbit

$u_{k} \quad$ Angular rate of change of the velocity orientation relative to the flow of particle $k$

$u_{\max }$ Maximum turn rate

$\gamma_{k} \quad$ Orientation of the inertial velocity of particle $k$

$\nu_{k} \quad$ Angular rate of change of the inertial-velocity orientation for particle $k$

$\omega_{0} \quad$ Constant angular rate

$\psi_{k} \quad$ Time-phase of particle $k$

$\theta_{k} \quad$ Orientation of the velocity of particle $k$ relative to the flow

Subscript

$j, k \quad$ Particle and phase indices, $1, \ldots, N$

\footnotetext{
${ }^{*}$ Graduate student, Department of Aerospace Engineering; cammykai@yahoo.com. AIAA Student Member.

${ }^{\dagger}$ Assistant Professor, Department of Aerospace Engineering; dpaley@umd.edu. AIAA Senior Member.
} 


\section{Introduction}

Multi-vehicle coordination improves the information gathered for autonomous vehicle missions. Coordinated vehicles can provide persistent coverage, maintain continual target tracks and provide wide-area sampling over a variety of domains. The benefits of cooperating vehicles has prompted much research in developing stable, decentralized control algorithms for a variety of autonomous platforms and configurations. ${ }^{1-10}$ These algorithms have been adapted for use in numerous applications including aerobiological sampling, ${ }^{11}$ gathering in situ measurements of severe storms, ${ }^{12}$ path planning of autonomous underwater gliders, ${ }^{13}$ and environmental boundary tracking. ${ }^{14}$ A particular application highlighted is the coordinated encirclement of a maneuvering target. ${ }^{5,6,10,15}$

One obstacle limiting the performance of existing control algorithms is the presence of an external flowfield that may represent a significant fraction of a vehicle's velocity. Some existing algorithms support operations in spatially uniform flowfields. ${ }^{16,17}$ However, the authors are not aware of any existing theoretically justified motion-coordination algorithms for spatially and temporally varying flowfields. This problem is partially addressed here by providing decentralized control algorithms for motion coordination in a moderate, timevarying flowfield (i.e., between $10 \%$ and $99 \%$ of platform speed). This work extends previous work in which decentralized control algorithms are presented for a time-invariant flowfield. ${ }^{18,19}$ Strong flowfields are not addressed here (i.e., having speed greater than the platform speed relative to the flow); this subject is being studied as part of ongoing research.

This paper builds upon prior research on cooperative control for autonomous vehicles. Frew et al. showed two vehicles collaborating to encircle a target while maintaining a constant angular separation. ${ }^{5}$ The separation is preserved with a variable-speed controller while the distance to the target is regulated by following Lyapunov-based guidance vector fields. The vector fields steer the vehicles in a loiter circle at a desired standoff radius. This approach was expanded by Summers et al. who relax the assumption that the flowfied or target velocity is known and allow cooperative control of two or more vehicles. ${ }^{6}$ However, a variable-speed control design has the potential disadvantage of being fuel inefficient. ${ }^{6}$

Constant-speed controllers were provided by Sepulchre et al. for a flow-free environment, maintaining a uniform separation of vehicles traveling around a circle using decentralized steering control. In the absence of a flowfield, the vehicles can be equally spaced both in space and time. Maintaining an equal angular separation in the presence of a flowfield may not be possible with a unit-speed vehicle model. Klein et al. proposed a temporal-spacing control for tracking a constant-velocity target; ${ }^{15}$ the algorithm placed a restriction on the number of vehicles in the formation. Kingston proposed a sliding-mode algorithm that would allow for an unlimited number of cooperating vehicles to encircle moving targets in a steady, uniform external flowfield. ${ }^{8}$ Paley et al. provided a Lyapunov-based control design to encircle a centroid in a timeinvariant flowfield. ${ }^{19}$ Techy et al. also showed that stabilization to circular formations in a flowfield can be extended to stabilization of a convex loop..$^{20,21}$

As in prior work, ${ }^{19}$ each autonomous vehicle is modeled as a Newtonian particle that travels at unit speed relative to the flow. The flowfield may be spatially or temporally varying, but its magnitude is assumed to be less than the speed of the particle. This ensures that each particle will always maintain forward progress over ground. The particle is subject to a steering control that is justified using Lyapunov-based analysis, the invariance principle, and an invariance-like principle for non-autonomous systems. (The latter is invoked in the analysis of the closed-loop particle model with a time-varying flowfield.)

The steering control is designed to drive the particles into a circular formation centered on either an arbitrary location or a specific target. By accounting for target acceleration, one can follow targets as they execute realistic maneuvers. Additionally, target encirclement is presented for a time-splay configuration in a time-varying, uniform flowfield. A time-splay configuration regulates the temporal spacing between particles. Regulating the spatial separation between particles is a first step toward collision avoidance. However, it does not guarantee separation of the particles while they transition to the configuration. Overall collision avoidance is not addressed in this paper and is left as a future topic of study.

The contribution of this paper is the synthesis of theoretically justified control algorithms to cooperatively stabilize vehicles to the following formations: 1) a circular formation in an unknown spatially uniform flowfield, and 2) a time-splay configuration in a unknown time-varying, spatially uniform flowfield. By assuming a known flowfield it is shown that these controllers operate under a restricted turn rate. These results are extended to an unknown, spatially uniform flowfield in simulations. Accelerating-frame dynamics are provided so that the control algorithms may be applied to encircling maneuvering targets. Simulation results demonstrate the utility of these control algorithms. 
The paper proceeds as follows. Section II presents the Newtonian-particle model used to describe the motion of a fleet of autonomous vehicles in an external flowfield. Section III discusses control laws for use in an external flow when aspects of the flowfield are unknown. Section IV provides algorithms to stabilize circular formations of in a spatially uniform or rotating flowfield with a turn-rate constraint. Section $V$ shows the equations of motion for an accelerating frame and provides performance results for circular configurations in a variety of velocities representing a maneuvering target. Section $\mathrm{VI}]$ summarizes the results and highlights some ongoing work.

\section{Dynamic Model of Vehicles in a Flowfield}

Each unmanned vehicle is modeled as a planar, self-propelled particle moving at unit speed relative to a spatially and temporally variable flowfield. This model has been adopted frequently in previous works for a flow-free environment. ${ }^{1,2}$ Each particle is steered by a control force that is perpendicular to the velocity relative to the flow. The steering control, denoted by $u$, is constrained for many autonomous vehicles. For example, with an unmanned aerial vehicle (UAV) one factor restricting the steering control is the bank angle of the aircraft. Since there is a finite limit to the degree to which a UAV can bank, the steering control is bounded. To model this effect a bound is placed on the turn-rate by saturating $u$.

The positions of $\mathrm{N}$ individual particles are denoted as $r_{k}$, where $k \in\{1, \ldots, N\}$. The inertial velocity of the $k$ th particle is denoted by $\dot{r}_{k}$. The particles do not accelerate tangentially to their path and thus move with unit velocity $e^{i \theta_{k}}$ relative to the flowfield. The flowfield at $r_{k}$ and time $t$ is denoted by $f_{k}(t)=f\left(r_{k}, t\right)$. The equations of motion for particle $k$ are

$$
\begin{aligned}
& \dot{r}_{k}=e^{i \theta_{k}}+f_{k}(t) \\
& \dot{\theta}_{k}=\operatorname{sat}\left(u_{k} ; u_{\max }\right),
\end{aligned}
$$

where $u_{\max }>0$ and

$$
\operatorname{sat}\left(\mathrm{u}_{\mathrm{k}} ; \mathrm{u}_{\max }\right)= \begin{cases}-u_{\max } & u_{k}<-u_{\max } \\ u_{k} & -u_{\max } \leq u_{k} \leq u_{\max } \\ u_{\max } & u_{k}>u_{\max } .\end{cases}
$$

For a vehicle with an unbounded turning rate, $u_{\max }=\infty$ and the model (1) becomes

$$
\begin{aligned}
& \dot{r}_{k}=e^{i \theta_{k}}+f_{k}(t) \\
& \dot{\theta}_{k}=u_{k} .
\end{aligned}
$$

Let $\gamma_{k}=\arg \left(\dot{r}_{k}\right)$ equal the orientation of the inertial velocity of the $k$ th particle and $s_{k}(t)=s\left(t, r_{k}, \theta_{k}\right)=$ $\left|\dot{r}_{k}\right|$ denote its magnitude. The particle model without the turn-rate constraint is equivalent to ${ }^{19}$

$$
\begin{aligned}
\dot{r}_{k} & =s_{k}(t) e^{i \gamma_{k}} \\
\dot{\gamma}_{k} & =\nu_{k},
\end{aligned}
$$

where $\nu_{k}$ is the angular rate of change of the inertial-velocity orientation. Note, it is required that $\left|f_{k}(t)\right|<1$ for all $k, t$, to ensure $s_{k}(t)>0$. This assumption guarantees that the particles will always exhibit forward motion in an inertial reference frame.

Section III discusses stabilization of circular formation in an unknown flowfield using the unconstrained particle model (4). Section IV invokes the turn-rate constrained model (1).

Note, we require $\left|f_{k}(t)\right|<1 \forall k, t$, which ensures $\left|s_{k}(t)\right|>0$. This assumption guarantees that each particle always exhibits forward motion in an inertial coordinate frame.

The controller design focuses on obtaining a suitable $\nu_{k} ; u_{k}$ is seen as the low-level controller used as input for an autonomous vehicle and should be recoverable from $\nu_{k}$. The following relationshin ${ }^{a}$ can be defined by the definitions of $\theta_{k}$ and $\gamma_{k}:{ }^{19}$

$$
\begin{aligned}
\sin \theta_{k} & =s_{k}(t) \sin \gamma_{k}-\left\langle f_{k}(t), i\right\rangle \\
\cos \theta_{k} & =s_{k}(t) \cos \gamma_{k}-\left\langle f_{k}(t), 1\right\rangle,
\end{aligned}
$$

\footnotetext{
${ }^{\mathrm{a}}$ We use the inner product $\langle x, y\rangle=\operatorname{Re}\{\bar{x} y\}$, where $x, y \in \mathbb{C}$ and $\bar{x}$ is the complex conjugate of $x$.
} 
which gives

$$
\tan \gamma_{k}=\frac{\sin \theta_{k}+\left\langle f_{k}(t), i\right\rangle}{\cos \theta_{k}+\left\langle f_{k}(t), 1\right\rangle}
$$

Differentiating (7) with respect to time and substituting in equations (5) and (6) yields

$$
\begin{aligned}
\dot{\gamma}_{k} & =\left(\cos \theta_{k} \cos \gamma_{k}+\sin \theta_{k} \sin \gamma_{k}\right) s_{k}^{-1}(t) \dot{\theta}_{k}+\left\langle\dot{f}_{k}, i\right\rangle s_{k}^{-1}(t) \cos \gamma_{k}-\left\langle\dot{f}_{k}, 1\right\rangle s_{k}^{-1}(t) \sin \gamma_{k} \\
& =\left(1-s_{k}^{-1}(t)\left\langle e^{i \gamma_{k}}, f_{k}(t)\right\rangle\right) u_{k}+s_{k}^{-1}(t)\left\langle i e^{i \gamma_{k}}, \dot{f}_{k}\right\rangle \triangleq \nu_{k},
\end{aligned}
$$

with

$$
\dot{f}_{k}=\frac{\partial f_{k}}{\partial r_{k}} \dot{r}_{k}+\frac{\partial f_{k}}{\partial t}
$$

Solving for $u_{k}(t)$ provides the steering control as a function of $\nu_{k}$ :

$$
u_{k}(t)=\frac{s_{k}(t) \nu_{k}-\left\langle i e^{i \gamma_{k}}, \dot{f}_{k}\right\rangle}{s_{k}(t)-\left\langle e^{i \gamma_{k}}, f_{k}(t)\right\rangle}
$$

The preceding equation is well-defined everywhere. Because the denominator satisfies $s_{k}(t)-\left\langle e^{i \gamma_{k}}, f_{k}(t)\right\rangle \geq$ $s_{k}(t)-\left|f_{k}(t)\right|>0 .{ }^{19}$

\section{Stabilization of Circular Formations in an Unknown Time-Varying Flowfield}

This section introduces control laws that stabilize a circular formation in an estimated uniform flowfield whose direction may be rotating in time. Section III.A and III.B present methods to stabilize circular formations with an arbitrary or prescribed center point in a uniform, time-invariant flowfield. In Section III.C a time-splay formation is stabilized in a uniform, rotating flow. It is assumed that each particle knows its measured position, $r_{k}$, and orientation, $\theta_{k}$, although a dynamic estimate of position, $\hat{r}_{k}$, is used to estimate the flow.

With the estimated flow, $\hat{f}_{k}(t)=\hat{f}\left(t, r_{k}\right)$, the estimated inertial velocity obeys

$$
\begin{aligned}
\dot{\hat{r}}_{k} & =\hat{s}_{k}(t) e^{i \hat{\gamma}_{k}} \\
\dot{\hat{\gamma}}_{k} & =\nu_{k},
\end{aligned}
$$

where $\hat{s}_{k}(t)$ and $\hat{\gamma}_{k}$ are the magnitude and phase, respectively, of the estimated inertial velocity for particle $k$.

\section{III.A. Circular Formation with an Arbitrary Center}

In this section a control law is developed to drive particles in a circular formation about an arbitrary, fixed point in the presence of an estimated, uniform, time-invariant flowfield. The control law works by dynamically estimating the flow and using the estimate in the control law.

Let $e_{1, k}=\hat{r}_{k}-r_{k}$ and $e_{2, k}=\hat{f}_{k}-f_{k}$ denote the estimation errors for particle $k$. Consider the estimator dynamics

$$
\begin{aligned}
\dot{\hat{r}}_{k} & =e^{i \theta_{k}}+\hat{f}_{k}-K_{1}\left(\hat{r}_{k}-r_{k}\right) \\
\dot{\hat{f}}_{k} & =-K_{2}\left(\hat{r}_{k}-r_{k}\right) .
\end{aligned}
$$

This yields

$$
\begin{aligned}
& \dot{e}_{1, k}=e^{i \theta_{k}}+\hat{f}_{k}-K_{1}\left(\hat{r}_{k}-r_{k}\right)-e^{i \theta_{k}}-f_{k}=-K_{1} e_{1, k}+e_{2, k} \\
& \dot{e}_{2, k}=-K_{2}\left(\hat{r}_{k}-r_{k}\right)=-K_{2} e_{1, k} .
\end{aligned}
$$

In matrix form, the estimator dynamics for particle $k$ are

$$
\left[\begin{array}{c}
\dot{e}_{1, k} \\
\dot{e}_{2, k}
\end{array}\right]=\underbrace{\left[\begin{array}{ll}
-K_{1} & 1 \\
-K_{2} & 0
\end{array}\right]}_{\triangleq A}\left[\begin{array}{l}
e_{1, k} \\
e_{2, k}
\end{array}\right] .
$$

$$
4 \text { of } 20
$$


Lemma 1. Choosing gains $K_{2}>0$ and $K_{1}=2 \sqrt{K_{2}}>0$ in the error dynamics 13) exponentially stabilizes the origin $e_{1, k}=e_{2, k}=0 \forall k$.

Proof. The eigenvalues of $A$ are $\lambda=\left(-K_{1} \pm \sqrt{K_{1}^{2}-4 K_{2}}\right) / 2$. Choosing $K_{1}=2 \sqrt{K_{2}}$ results in $\lambda=-K_{1}$ with multiplicity two.

The following is a result of Lemma 1 .

Lemma 2. The matrix A defined in (13) is negative definite and the quadratic form

$$
Q_{k}(A)=\left[\begin{array}{ll}
e_{1, k} & e_{2, k}
\end{array}\right]\left[\begin{array}{cc}
-K_{1} & 1 \\
-K_{2} & 0
\end{array}\right]\left[\begin{array}{c}
e_{1, k} \\
e_{2, k}
\end{array}\right]=-K_{1} e_{1, k}^{2}-K_{2} e_{1, k} e_{2, k}+e_{1, k} e_{2, k} \leq 0
$$

is equal to zero only when $e_{1, k}=e_{2, k}=0$ for $k \in\{1, \ldots, N\}$.

Let $\hat{c}_{k}$ be the estimated center,

$$
\hat{c}_{k}=\hat{r}_{k}+\omega_{0}^{-1} i e^{i \hat{\gamma}_{k}}
$$

Differentiating (15) with respect to time we find a steering control $\nu_{k}$ that allows us to drive a single particle around a circle at the estimated center point. We have

$$
\dot{\hat{c}}_{k}=\hat{s}_{k} e^{i \hat{\gamma}_{k}}-\omega_{0}^{-1} e^{i \hat{\gamma}_{k}} \nu_{k}=\left(\hat{s}_{k}-\omega_{0}^{-1} \nu_{k}\right) e^{i \hat{\gamma}_{k}} .
$$

Control $\nu_{k}=\omega_{0} \hat{s}_{k}$ ensures that the circle center is fixed, $\dot{\hat{c}}_{k}=0$, and particle $k$ will travel in a circle.

Consider the candidate Lyapunov function

$$
\hat{S}(\hat{\boldsymbol{r}}, \hat{\gamma}) \triangleq \frac{1}{2}\langle\hat{\boldsymbol{c}}, P \hat{\boldsymbol{c}}\rangle+\frac{1}{2}\left(\left\|\boldsymbol{e}_{1}\right\|^{2}+\left\|\boldsymbol{e}_{2}\right\|^{2}\right)
$$

where $\boldsymbol{e}_{1}=\left[e_{1,1}, e_{1,2}, \ldots, e_{1, N}\right]^{T}$ and $\boldsymbol{e}_{2}=\left[e_{2,1}, e_{2,2}, \ldots, e_{2, N}\right]^{T} . \hat{r}, \hat{\gamma}$ and $\hat{c}$ are the vectors of position, orientation and center points for all $k$ particles. $P$ is the $N \times N$ projection matrix

$$
P=\operatorname{diag}\{\mathbf{1}\}-\frac{1}{N} \mathbf{1 1}^{T},
$$

which is equivalent to the Laplacian matrix of an all-to-all communication topology. ${ }^{9} \hat{S}$ is equal to zero when $\hat{\boldsymbol{c}}=c_{0} \mathbf{1}, c_{0} \in \mathbb{C}$, and the estimation errors are zero.

The time derivative of $\hat{S}$ along solutions of 11 and 13 is

$$
\begin{aligned}
\dot{\hat{S}} & =\sum_{k=1}^{N}\left(\left\langle\dot{\hat{c}}_{k}, P_{k} \hat{\boldsymbol{c}}\right\rangle+\dot{e}_{1, k} e_{1, k}+\dot{e}_{2, k} e_{2, k}\right) \\
& =\sum_{k=1}^{N}[\left\langle e^{i \hat{\gamma}_{k}}, P_{k} \hat{\boldsymbol{c}}\right\rangle\left(\hat{s}_{k}(t)-\omega_{0}^{-1} \nu_{k}\right)+\underbrace{e_{1, k}\left(-K_{1} e_{1, k}+e_{2, k}\right)+e_{2, k}\left(-K_{2} e_{1, k}\right)}_{=Q_{k}(A)}] .
\end{aligned}
$$

The following theorem extends [22, Theorem 1] to the case of an unknown, uniform, time-invariant flowfield.

Theorem 1. Let $f_{k}(t)=\beta \in \mathbb{R}$, where $|\beta|<1$, be an unknown time-invariant flowfield. Also, let $\hat{r}_{k}$ and $\hat{f}_{k}$ evolve according to (12) with $K_{2}>0$ and $K_{1}=2 \sqrt{K_{2}}$. Choosing the control

$$
\nu_{k}=\omega_{0}\left(\hat{s}_{k}(t)+K\left\langle P_{k} \hat{\boldsymbol{c}}, e^{i \hat{\gamma}_{k}}\right\rangle\right), K>0,
$$

forces convergence of solutions of model (11) to the set of a circular formations with radius $\left|\omega_{0}\right|^{-1}$ and direction determined by the sign of $\omega_{0}$.

Proof. Substituting 20 into 19 shows that the time-derivative of the potential $\hat{S}(\hat{\boldsymbol{r}}, \hat{\gamma})$ satisfies

$$
\dot{\hat{S}}=\sum_{k=1}^{N}\left(-K\left\langle P_{k} \hat{\boldsymbol{c}}, e^{i \hat{\gamma}_{k}}\right\rangle^{2}+Q_{k}(A)\right) \leq 0 .
$$


Using the invariance principle, all of the solutions of (4) with controller 20 converge to the largest invariant set where

$$
-K\left\langle P_{k} \hat{\boldsymbol{c}}, e^{i \hat{\gamma}_{k}}\right\rangle^{2}+Q_{k}(A)=0, \forall k
$$

By Lemma 2 condition 22 is satisfied only when both $Q_{k}(A)=0$ and $\left\langle P_{k} \hat{\boldsymbol{c}}, e^{i \hat{\gamma}_{k}}\right\rangle=0$ independently. $Q_{k}(A)=0$ implies that estimated values $\hat{r}_{k}$ and $\hat{f}_{k}$ equal the measured values, $r_{k}$ and $f_{k}$. Values $\hat{\gamma}_{k}$ and $\hat{s}_{k}(t)$ are functions of $\hat{f}_{k}$ and $\theta_{k}$. This implies that $\hat{\gamma}_{k}$ and $\hat{s}_{k}(t)$ approach their measured values and, by (15), $\hat{c}_{k}$ converges to $c_{k}$. The condition, $\left\langle P_{k} \hat{\boldsymbol{c}}, e^{i \hat{\gamma}_{k}}\right\rangle=0$ is satisfied for all $k$ only when $P_{k} \hat{\boldsymbol{c}}$ is constant and equal to zero. Since the null space of $P$ is spanned by 1 this implies $\hat{c}_{k}=\hat{c}_{j} \forall k, j$. In this set, control (20) evaluates to $\nu_{k}=\omega_{0} \hat{s}_{k}(t)$ and $\dot{\hat{c}}_{k}=0$ which implies that each particle converges to circular motion around the same fixed center.

\section{III.B. Circular Formation with a Prescribed Center}

Under the control 20 the center of the circular formation depends only on the initial conditions of the particles and the flowfield. By introducing a symmetry-breaking virtual particle (indexed by $k=0$ ) we can prescribe a center point for the formation. ${ }^{19}$ Consider the augmented potential $\tilde{S}(\hat{\boldsymbol{r}}, \hat{\gamma})=\hat{S}(\hat{\boldsymbol{r}}, \hat{\gamma})+S_{0}(\hat{\boldsymbol{r}}, \hat{\gamma})$, where

$$
S_{0}(\hat{\boldsymbol{r}}, \hat{\gamma})=\frac{1}{2} \sum_{j=1}^{N} a_{j 0}\left|\hat{c}_{j}-c_{0}\right|^{2}
$$

and $\hat{c}_{k}$ is defined by (15). $a_{k 0}=1$ if particle $k$ is informed of the reference center, $c_{0}$, and $a_{k 0}=0$ otherwise. Taking the time-derivative of (23) along solutions of (11) and (13) gives

$$
\dot{\tilde{S}}=\sum_{j=1}^{N}[\left(\left\langle e^{i \hat{\gamma}_{j}}, P_{j} \hat{\boldsymbol{c}}\right\rangle+a_{j 0}\left\langle e^{i \hat{\gamma}_{j}}, \hat{c}_{j}-c_{0}\right\rangle\right)\left(\hat{s}_{j}(t)-\omega_{0}^{-1} \nu_{j}\right)+\underbrace{e_{1, j}\left(-K_{1} e_{1, j}+e_{2, j}\right)+e_{2, j}\left(-K_{2} e_{1, j}\right)}_{=Q_{j}(A)}] .
$$

Theorem 2. Let $f_{k}(t)=\beta \in \mathbb{R}$, where $|\beta|<1$, be an unknown time-invariant flow. Also, let $\hat{r}_{k}$ and $\hat{f}_{k}$ evolve according to (12) with $K_{2}>0$ and $K_{1}=2 \sqrt{K_{2}}$. Choosing the control

$$
\nu_{k}=\omega_{0}\left(\hat{s}_{k}(t)+K\left(\left\langle e^{i \hat{\gamma}_{k}}, P_{k} \hat{\boldsymbol{c}}\right\rangle+a_{k 0}\left\langle e^{i \hat{\gamma}_{k}}, \hat{c}_{k}-c_{0}\right\rangle\right)\right), K>0,
$$

forces convergence of solutions of model (11) to the set of a circular formation centered at $c_{0}$ with radius $\left|\omega_{0}\right|^{-1}$ and direction determined by the sign of $\omega_{0}$.

Proof. Substituting the controller (25) into (24) shows that the time-derivative of the augmented potential $\tilde{S}(\hat{\boldsymbol{r}}, \hat{\gamma})$ satisfies

$$
\dot{\tilde{S}}=\sum_{j=1}^{N}\left(\left(-K\left\langle e^{i \hat{\gamma}_{j}}, P_{j} \hat{\boldsymbol{c}}\right\rangle+a_{j 0}\left\langle e^{i \hat{\gamma}_{j}}, \hat{c}_{j}-c_{0}\right\rangle\right)^{2}+Q_{j}(A)\right) \leq 0 .
$$

According to the invariance principle all solutions of 4 with controller 25 converge to the set $\{\dot{\tilde{S}}=0\}$ where

$$
\left\langle P_{j} \hat{\boldsymbol{c}}, e^{i \hat{\gamma}_{j}}\right\rangle^{2}+a_{j 0}\left\langle e^{i \hat{\gamma}_{j}}, \hat{c}_{j}-c_{0}\right\rangle+Q_{k}(A)=0, \forall j
$$

By Lemma $2 Q_{k}(A)=0$ only when the estimated $\hat{r}_{k}$ and $\hat{f}_{k}$ values have converged to their corresponding real values. The convergence of these estimated values also implies that $\hat{s}_{k}, \hat{\gamma}_{k}$ and $\hat{c}_{k}$ converge to their real values. The remaining terms in (26), $\left\langle P_{j} \hat{\boldsymbol{c}}, e^{i \hat{\gamma}_{j}}\right\rangle^{2}+a_{j 0}\left\langle e^{i \gamma_{j}}, \hat{c}_{j}-c_{0}\right\rangle$, must be equal to zero independently of $Q_{k}(A)$. Following the proof of [19, Corollary 1] there are two potential cases to consider, first if $a_{k 0}=0$ for at least one particle or second if $a_{k 0}=1, \forall k$. The first case implies that $P_{k} \hat{\boldsymbol{c}}=0$ for at least one particle $k$, indicating that $\hat{\boldsymbol{c}}$ is in the span of $\mathbf{1}$ and $\hat{c}_{k}=\hat{c}_{j}$ for all pairs $k$ and $j$. Putting $P \hat{\boldsymbol{c}}=0$ back into (26) further implies that $\hat{c}_{k}=c_{0}$ and the controller 25) becomes $\nu_{k}=\hat{s}_{k} \omega_{0}$. The trivial case of $a_{k 0}=1, \forall k$ is addressed in [19, Corollary 1]. All particles in the set $\{\dot{\tilde{S}}=0\}$ will travel in a circular formation with radius $\left|\omega_{0}\right|^{-1}$ and center point $c_{0}$. 


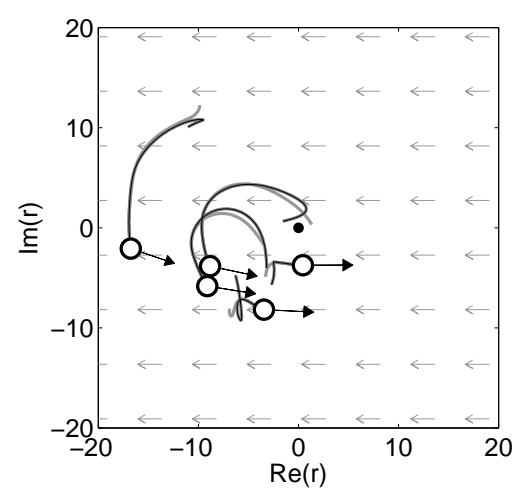

(a) $t=20 \mathrm{~s}$

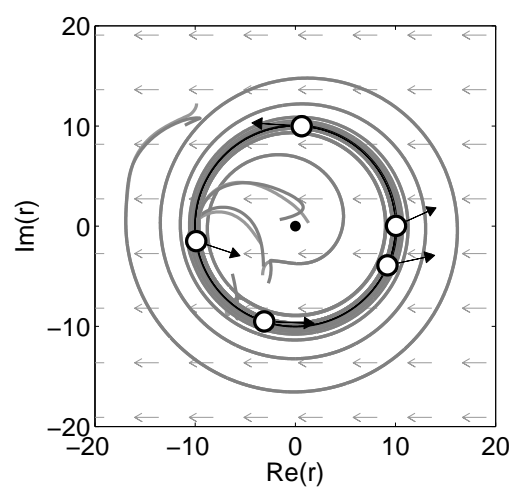

(b) $t=1000 \mathrm{~s}$

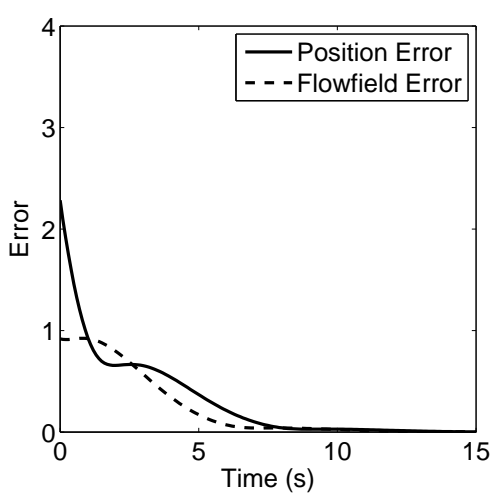

(c) Error dynamics, $k=3$

Figure 1. Stabilization of circular formation in uniform flowfield $f=-0.8$ with a prescribed center point $c_{0}=0$.

Figure 1 illustrates Theorem 2 with estimator gains $K_{2}=0.2$ and $K_{1}=2 \sqrt{K_{2}}=0.894$ and a uniform flowfield $f=-0.8$. Figures $1(\mathrm{a})$ and $1(\mathrm{~b})$ show tracks of the estimated (darker track) and actual (lighter track) particle positions at 20 and 1000 seconds respectively, as they converge to a circular formation about the prescribed center point, $c_{0}=0$. Figure $1(\mathrm{c})$ shows convergence to zero of estimator errors in the position and the flow for particle $k=3$.

\section{III.C. Time-splay Formation with a Rotating Flowfield}

In this section a control law is derived to stabilize particles to a time-splay formation in an estimated, spatially invariant, rotating flowfield. (A rotating flowfield is defined to be a uniform flowfield whose direction is rotating in time.) Without speed control it may not be possible to regulate the spatial separation of particles, but it is possible to regulate the temporal separation in an estimated flowfield as shown next.

Given a uniform time-varying flowfield $f=\eta_{0} e^{i \Omega t}$, choose $e_{1, k}=\hat{r}_{k}-r_{k}$ and $e_{2, k}=\hat{f}_{k}-f_{k}$. The rotational rate, $\Omega$, is assumed to be known; the flow speed, $\eta_{0}$, and initial orientation are unknown. Consider the estimator dynamics

$$
\begin{aligned}
& \dot{\hat{r}}_{k}=e^{i \theta_{k}}+\hat{f}_{k}-K_{1}\left(\hat{r}_{k}-r_{k}\right) \\
& \dot{\hat{f}}_{k}=-K_{2}\left(\hat{r}_{k}-r_{k}\right)+\Omega i \hat{f}_{k} .
\end{aligned}
$$

Taking the derivative of the error and plugging in 27) yields

$$
\begin{aligned}
& \dot{e}_{1, k}=e^{i \theta_{k}}+\hat{f}_{k}-K_{1}\left(\hat{r}_{k}-r_{k}\right)-e^{i \theta_{k}}-f_{k}=-K_{1} e_{1, k}+e_{2, k} \\
& \dot{e}_{2, k}=-K_{2} e_{1, k}+\Omega i e_{2, k} .
\end{aligned}
$$

The estimator dynamics for particle $k$ are

$$
\left[\begin{array}{c}
\dot{e}_{1, k} \\
\dot{e}_{2, k}
\end{array}\right]=\underbrace{\left[\begin{array}{cc}
-K_{1} & 1 \\
-K_{2} & \Omega i
\end{array}\right]}_{\triangleq B}\left[\begin{array}{c}
e_{1, k} \\
e_{2, k}
\end{array}\right] .
$$

Lemma 3. Choosing gains $K_{1}>|\Omega|$ and $K_{2}=\left(K_{1}^{2}-\Omega^{2}\right) / 4$ in the error dynamics (28) exponentially stabilizes the origin $e_{1, k}=e_{2, k}=0$ for $k=[1, \ldots, N]$.

Proof. The real parts of the eigenvalues of $B$ (see Appendix VI) are

$$
\operatorname{Re}(\lambda)=\frac{-K_{1}}{2} \pm \frac{\sqrt{\sqrt{\left(K_{1}^{2}-\Omega^{2}-4 K_{2}\right)^{2}+4 K_{1}^{2} \Omega^{2}}+\left(K_{1}^{2}-\Omega^{2}-4 K_{2}\right)}}{2 \sqrt{2}} .
$$

Letting $K_{1}>|\Omega|$ and $K_{2}=\left(K_{1}^{2}-\Omega^{2}\right) / 4$ results in $\operatorname{Re}(\lambda)=\left(-K_{1} \pm \sqrt{K_{1} \Omega}\right) / 2 . \quad K_{1}>|\Omega|$, which implies $\operatorname{Re}(\lambda)<0$. 
The following result is a consequence of Lemma 3

Lemma 4. Matrix $B$ defined in (28) is negative definite and the quadratic form

$$
Q_{k}(B) \triangleq\left[e_{1, k} e_{2, k}\right]\left[\begin{array}{cc}
-K_{1} & 1 \\
-K_{2} & \Omega i
\end{array}\right]\left[\begin{array}{l}
e_{1, k} \\
e_{2, k}
\end{array}\right]=-K_{1} e_{1, k}^{2}-K_{2} e_{1, k} e_{2, k}+e_{1, k} e_{2, k}+\Omega i e_{2, k}^{2} \leq 0
$$

is only equal to zero when $e_{1, k}=e_{2, k}=0 \forall k$.

From $(16)$ shows that $\nu_{k}=\omega_{0} \hat{s}_{k}$ drives particle $k$ in a fixed circle of radius $\left|\omega_{0}\right|^{-1}$. Consider the change of variables $\hat{\gamma}_{k}^{\prime}=\hat{\gamma}_{k}-\Omega t$, which implies

$$
\dot{\hat{\gamma}}_{k}^{\prime}=\dot{\hat{\gamma}}_{k}-\Omega=\omega_{0} \hat{s}_{k}-\Omega
$$

The estimated inertial speed, $\hat{s}_{k}$, which does not depend explicitly on time, is ${ }^{22}$

$$
\hat{s}_{k}=\eta_{0} \cos \hat{\gamma}_{k}^{\prime}+\sqrt{\left(1-\eta_{0}^{2} \sin ^{2} \hat{\gamma}_{k}^{\prime}\right)}
$$

For the ensuing calculations to be nonsingular, we require that (31) not have a fixed point, according to the following Lemma.

Lemma 5. Choosing

$$
\left|\omega_{0}\right|>\frac{\operatorname{sgn}\left(\omega_{0}\right) \Omega}{\left(1-\left|\eta_{0}\right|\right)} \text { or }\left|\omega_{0}\right|<\frac{\operatorname{sgn}\left(\omega_{0}\right) \Omega}{\left(1+\left|\eta_{0}\right|\right)}
$$

ensures that (31) does not have a fixed point.

Proof. Requiring $\omega_{0} \hat{s}_{k}(t)-\Omega \neq 0$ implies

$$
\min _{\hat{\gamma}_{k}^{\prime}} \hat{s}\left(\hat{\gamma}_{k}^{\prime}\right)>\frac{\Omega}{\omega_{0}}
$$

or

$$
\max _{\hat{\gamma}_{k}^{\prime}} \hat{s}\left(\hat{\gamma}_{k}^{\prime}\right)<\frac{\Omega}{\omega_{0}}
$$

The minimum of $\hat{s}\left(\hat{\gamma}_{k}^{\prime}\right)$ in 32 is $\hat{s}\left(\hat{\gamma}_{k}^{\prime}\right)=1-\left|\eta_{0}\right|$, which occurs at $\gamma_{k}^{\prime}=\pi$ if $\eta_{0}>0$ and $\gamma_{k}^{\prime}=0$ if $\eta_{0}<0$. Substituting $\hat{s}\left(\hat{\gamma}_{k}^{\prime}\right)=1-\left|\eta_{0}\right|$ into $\sqrt[34]{3}$ yields $\left|\omega_{0}\right|>\operatorname{sgn}\left(\omega_{0}\right) \Omega /\left(\left(1-\left|\eta_{0}\right|\right)\right)$. The maximum of $\hat{s}\left(\hat{\gamma}_{k}^{\prime}\right)$ in $\sqrt[32]{32}$ is $\hat{s}\left(\hat{\gamma}_{k}^{\prime}\right)=1+\left|\eta_{0}\right|$ which occurs at $\gamma_{k}^{\prime}=0$ if $\eta_{0}>0$ and $\gamma_{k}^{\prime}=\pi$ if $\eta_{0}<0$. Substituting $\hat{s}\left(\hat{\gamma}_{k}^{\prime}\right)=1+\left|\eta_{0}\right|$ into (35) yields $\operatorname{sgn}\left(\omega_{0}\right)\left|\omega_{0}\right|<\Omega /\left(\left(1+\left|\eta_{0}\right|\right)\right)$. Therefore, placing conditions (33) on $\left|\omega_{0}\right|$ ensures that (31) will not have a fixed point.

We use a time-phase quantity to regulate the temporal separation of the cooperating particles. ${ }^{19}$ This value is defined to $\mathrm{be}^{22}$

$$
\hat{\psi}_{k}=\frac{2 \pi}{T} \int_{0}^{\hat{\gamma}_{k}^{\prime}(t)} \frac{d \hat{\gamma}^{\prime}}{\omega_{0} \hat{s}\left(\hat{\gamma}^{\prime}\right)-\Omega},
$$

where $T$ is the period of a single particle's revolution. The time-derivative of $(36)$ is

$$
\dot{\hat{\psi}}_{k}=\frac{2 \pi}{T} \frac{\nu_{k}-\Omega}{\omega_{0} \hat{s}_{k}-\Omega} .
$$

The estimator dynamics (28) are used to stabilize a time-splay formation for a flowfield with known rotation rate $\Omega(t)$. Consider the Lyapunov function

$$
\hat{V}(\hat{\boldsymbol{r}}, \hat{\gamma})=\frac{1}{2}\langle\hat{\boldsymbol{c}}, P \hat{\boldsymbol{c}}\rangle+\frac{T}{2 \pi} U(\hat{\boldsymbol{\psi}})+\frac{1}{2}\left(\left\|\boldsymbol{e}_{1}\right\|^{2}+\left\|\boldsymbol{e}_{2}\right\|^{2}\right)
$$


where $\hat{\boldsymbol{c}}$ is the vector of estimated center points defined by 15.$) U(\hat{\boldsymbol{\psi}})$ is a smooth potential satisfying the rotational symmetry property, $U\left(\hat{\boldsymbol{\psi}}+\hat{\psi}_{0} \mathbf{1}\right)=U(\hat{\boldsymbol{\psi}}){ }^{2}$ Taking the time-derivative along solutions of 11 and (28) yields

$$
\begin{array}{r}
\dot{\hat{V}}=\sum_{k=1}^{N}\left[\left(\hat{s}_{k}(t)\left\langle e^{i \hat{\gamma}_{k}}, P_{k} \hat{\boldsymbol{c}}\right\rangle-\frac{\partial U}{\partial \hat{\psi}_{k}} \frac{\omega_{0} \hat{s}_{k}(t)}{\omega_{0} \hat{s}_{k}(t)-\Omega}\right)\left(\frac{\omega_{0} \hat{s}_{k}(t)-\nu_{k}}{\omega_{0} \hat{s}_{k}(t)}\right)+\right. \\
\underbrace{e_{1, k}\left(-K_{1} e_{1, k}+e_{2, k}\right)+e_{2, k}\left(-K_{2} e_{1, k}+\Omega i e_{2, k}\right)}_{=Q_{k}(B)}] .
\end{array}
$$

The following result extends [22, Theorem 3] to incorporate an unknown, spatially invariant, rotating flow.

Theorem 3. Let $f(t)=\eta_{0} e^{i \Omega t}$ be an unknown spatially invariant flowfield satisfying the condition $\left|\eta_{0}\right|<$ $1, \forall t$. Also, let $U(\hat{\psi})$ be a smooth, rotationally symmetric phase potential. Let $\hat{r}_{k}$ and $\hat{f}_{k}$ evolve according to 27) with $K_{1}>|\Omega|$ and $K_{2}=\left(K_{1}^{2}-\Omega^{2}\right) / 4$. Choosing the control

$$
\nu_{k}=\omega_{0} \hat{s}_{k}(t)\left(1+K\left(\hat{s}_{k}(t)\left\langle e^{i \hat{\gamma}_{k}}, P_{k} \hat{\boldsymbol{c}}\right\rangle-\frac{\partial U}{\partial \hat{\psi}_{k}} \frac{\omega_{0} \hat{s}_{k}(t)}{\omega_{0} \hat{s}_{k}(t)-\Omega}\right)\right), K>0,
$$

where $\omega_{0} \neq 0$ satisfies the constraint in Lemma 5, stabilizes the set of circular formations with radius $\left|\omega_{0}\right|^{-1}$ and direction determined by the sign of $\omega_{0}$ in which the time-phase arrangement is a critical point of $U(\hat{\boldsymbol{\psi}})$.

Proof. Using control (40) with potential (39) yields

$$
\dot{\hat{V}}=\sum_{k=1}^{N}\left(-K\left(\hat{s}_{k}(t)\left\langle e^{i \hat{\gamma}_{k}}, P_{k} \hat{\boldsymbol{c}}\right\rangle-\left(\frac{\partial U}{\partial \hat{\psi}_{k}} \frac{\omega_{0} \hat{s}_{k}(t)}{\omega_{0} \hat{s}_{k}(t)-\Omega}\right)\right)^{2}+Q_{k}(B)\right) \leq 0 .
$$

By an invariance-like principle [23, Theorem 8.4], solutions of 44 converge to the set $\{\dot{\hat{V}}=0\}$ for which

$$
-K\left(\hat{s}_{k}(t)\left\langle e^{i \hat{\gamma}_{k}}, P_{k} \hat{\boldsymbol{c}}\right\rangle-\frac{\partial U}{\partial \hat{\psi}_{k}} \frac{\omega_{0} \hat{s}_{k}(t)}{\omega_{0} \hat{s}_{k}(t)-\Omega}\right)^{2}+Q_{k}(B)=0, \forall k .
$$

Both terms in (41) must equal zero idenpendently. According to Lemma $4 Q_{k}(B)=0$ only when the estimated $\hat{r}_{k}$ and $\hat{f}_{k}$ values have converged to their corresponding measured values. The convergence of these estimated values along with the known value $\theta_{k}$ also implies that $\hat{s}_{k}(t), \hat{\gamma}_{k}$ and $\hat{c}_{k}$ converge to their measured values. The remaining quantities in (41) must independently be equal to zero,

$$
\hat{s}_{k}(t)\left\langle e^{i \hat{\gamma}_{k}}, P_{k} \hat{\boldsymbol{c}}\right\rangle-\frac{\partial U}{\partial \hat{\psi}_{k}} \frac{\omega_{0} \hat{s}_{k}(t)}{\omega_{0} \hat{s}_{k}(t)-\Omega}=0, \forall k .
$$

In this set, $\nu_{k}=\omega_{0} s_{k}(t)$, this is found by substituting 42 into control law (40). Thus each particle travels around a circle with a fixed center and radius $\left|\omega_{0}\right|^{-1}$. Substituting $\nu_{k}=\omega_{0} \hat{s}_{k}$ into 37 shows $\dot{\hat{\psi}}_{k}=2 \pi / T$, which implies that $U(\hat{\psi})$ is ,constant by its rotational symmetry property, ${ }^{2}$ and $\partial U / \partial \hat{\psi}_{k}=0 \forall k$. Constraint (42) reduces to

$$
\left\langle e^{i \gamma_{k}}, P_{k} c\right\rangle=0 .
$$

$\gamma_{k}$ is time-varying and (43) holds only if $P_{k} c$ is constant and equal to zero. Since the null space of $P$ is spanned by $1, P \boldsymbol{c}=0$ implies $c_{k}=c_{j} \forall k, j$. Since $\hat{V}$ decreases over the interval $[t, t+\delta] \forall t \geq 0$, for some $\delta>0$ the set of circular formations with radius $\left|\omega_{0}\right|^{-1}$ is uniformly, asymptotically stable [23, Theorem 8.5].

Figure 2 illustrates Theorem 3 . The simulation uses estimator gains $K_{1}=0.1$ and $K_{2}=\left(K_{1}^{2}-\Omega^{2}\right) / 4$. The rotating flowfield parameters are $\eta=0.5, \Omega=-0.01$, and $\omega_{0}=.1$ which satisfies Lemma 5 Figures 2(a) and 2(b) show tracks of the estimated (darker track) and actual (lighter track) particle positions at 20 and 3000 seconds respectively as they converge to a time-splay formation about the prescribed center point, $c_{0}=0$. Figure 2(c) shows convergence of the estimator errors to zero for a single particle, $k=3$. The initial conditions for all particles are set randomly. In this simulation the initial estimated flowfield started in the opposite direction as the actual flowfield causing the measured and estimated positions to temporarily diverge as seen by the peak in Figure 2(c). 


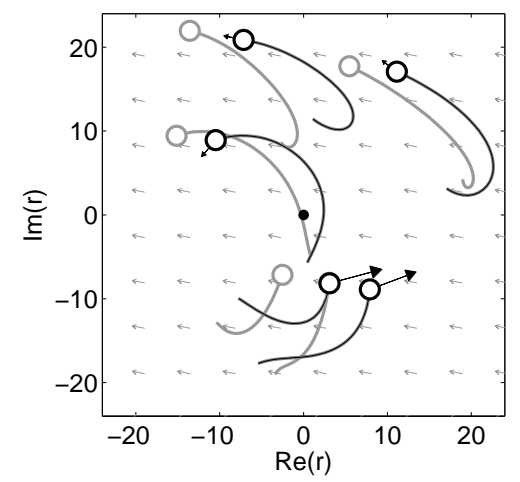

(a) $t=20 \mathrm{~s}$

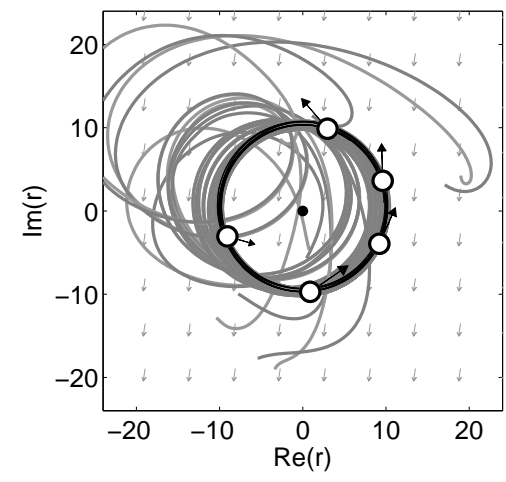

(b) $t=3000 \mathrm{~s}$

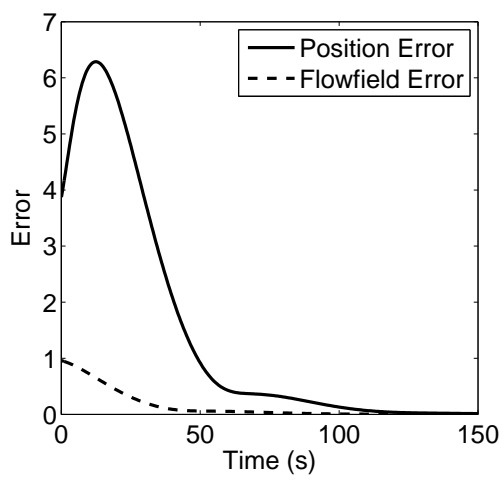

(c) Error Dynamics, $k=3$

Figure 2. Stabilization of a circular time-splay formation centered at $c_{0}=0$ in an estimated rotating flowfield, $f(t)=$ $\eta_{0} e^{i \Omega t}$, with $\eta_{0}=0.5$ and $\Omega=-0.01$.

\section{Formation Stabilization with Turn-Rate Constraint}

Physical restrictions on an autonomous vehicle often constrain the turn-rate control that may be applied to a system. In UAVs, a turn-rate constraint can result from the aircraft's maximum bank angle. ${ }^{24}$ In the previous sections this bound was relaxed, allowing for potentially unlimited turn-rate control. In this section it is shown that the previous results are valid even when there is a turn-rate constraint; the constraint imposes a lower bound on the feasible radius of a circular formation in a flowfield.

In terms of the inertial speed $s_{k}(t)$ and orientation $\gamma_{k}$, model (1) is equivalent to

$$
\begin{aligned}
& \dot{r}_{k}=s_{k}(t) e^{i \gamma_{k}} \\
& \dot{\gamma}_{k}=\operatorname{sat}\left(\nu_{k} ; \nu_{\max }\right),
\end{aligned}
$$

where $\nu_{\max } \triangleq \nu_{k}\left(u_{\max }\right)$ is a constraint on the steering control induced by the saturation on $u_{k}$. The relationship between $\nu_{\max }$ and $u_{\max }$ is defined in Section IV.A for a uniform, time-invariant flowfield and in Section IV.B for a uniform, rotating flowfield.

\section{IV.A. Turn-Rate Constraint in a Uniform, Time-Invariant Flowfield}

The following result establishes the maximum required turn rate for a particle to travel in a circle about a fixed center in a uniform, time-invariant flowfield. (Because of the flow the turn rate changes as the particle travels around the circle).

Lemma 6. Consider circular motion with radius $\left|\omega_{0}\right|^{-1}$ of a particle in a steady flow $f_{k}=\beta \in \mathbb{R}$. The maximum required turn rate is

$$
u_{\max } \triangleq \max _{\gamma_{k}} u\left(\gamma_{k}\right)=\left|\omega_{0}\right|(1+|\beta|)^{2}>0
$$

The maximum steering control is

$$
\nu_{\text {max }} \triangleq \nu\left(u_{\max }\right)=\left|\omega_{0}\right|(1+|\beta|) .
$$

Proof. Consider the particle model (1). The turn-rate control, $u_{k}$, for a spatially uniform, time-invariant flowfield is (see 10)

$$
u_{k}=\frac{\nu_{k}}{1-\beta s_{k}^{-1}(t) \cos \gamma_{k}} .
$$

Given a circular control $\nu_{k}=\omega_{0} s_{k}(t)$ with inertial speed

$$
s_{k}(t)=\beta \cos \gamma_{k}+\left(1-\beta^{2} \sin ^{2} \gamma_{k}\right)^{1 / 2},
$$




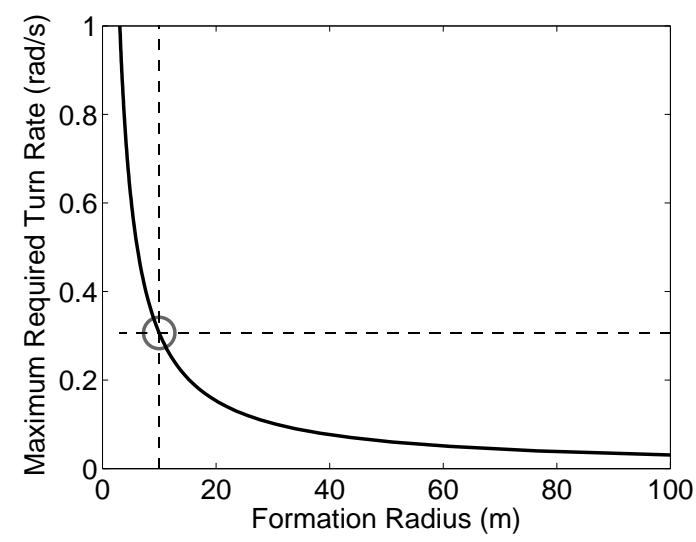

(a)

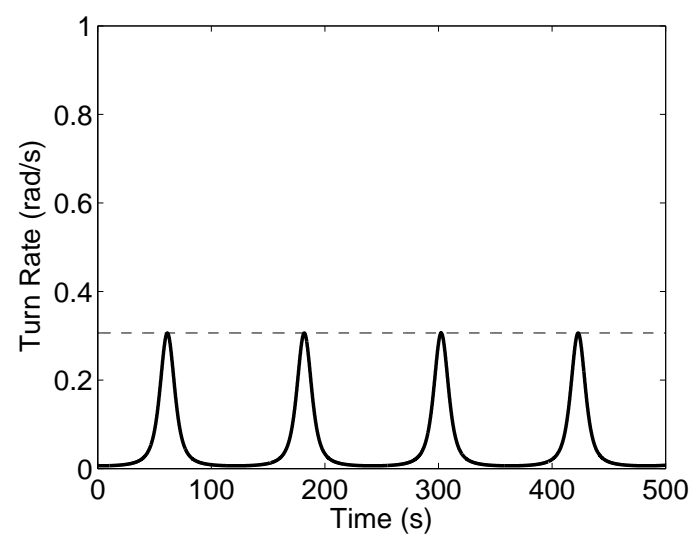

(b)

Figure 3. (a) Shows the maximum turn rate as a function of formation radius; (b) illustrates the turn rate as a function of time for a particle on a circle of radius $\left|\omega_{0}\right|^{-1}=10$.

the turn rate 47 is

$$
u_{k}=u\left(\gamma_{k}\right)=\frac{\omega_{0}\left(\beta \cos \gamma_{k}+\left(1-\beta^{2} \sin ^{2} \gamma_{k}\right)^{1 / 2}\right)^{2}}{\left(1-\beta^{2} \sin ^{2} \gamma_{k}\right)^{1 / 2}} .
$$

The maximum turn rate

$$
u_{\max }=\max _{\gamma_{k}} u\left(\gamma_{k}\right)=\left|\omega_{0}\right|(1+|\beta|)^{2}
$$

occurs when $\sin \gamma_{k}=0$ and $\beta \cos \gamma_{k}=|\beta|$, i.e., $\gamma_{k}=0$ if $\beta<0$ or $\gamma_{k}=\pi$ if $\beta>0$. Under these conditions (48) becomes $s_{k}(t)=1+|\beta|$. The controller $\nu_{k}$ is bounded through its relationship to $u_{\max }$. Substituting $s_{k}(t)=1+|\beta|$ and (50) into (47) gives

$$
\nu_{\max } \triangleq \nu\left(u_{\max }\right)=\frac{u_{\max }}{(1+|\beta|)^{2}}=\left|\omega_{0}\right|(1+|\beta|) .
$$

Figure $3(\mathrm{a})$ shows the maximum turn rate $u_{\max }$ as a function of the formation radius $\left|\omega_{0}\right|^{-1}$ for $\beta=0.75$. (The maximum turn rate is the value necessary to maintain a circular formation of radius $\left|\omega_{0}\right|^{-1}$ given a spatially uniform flow $\beta$ ). Figure 3(b) plots the turn rate for a single particle initialized with a random position and velocity as it converges under control $\nu_{k}=\omega_{0} s_{k}(t)$ to a circle of radius $\left|\omega_{0}\right|^{-1}=10$. Note that the particle stays at or below the maximum turn rate as it maintains a circle of the prescribed radius. The following result shows that, even if the steering control is saturated the circular-formation control law is still justified.

Theorem 4. Consider model (44) with $f_{k}=\beta \in \mathbb{R}, u_{\max }>0$, and $\nu_{\max }=\nu_{k}\left(u_{\max }\right)$. If $\omega_{0}$ satisfies

$$
\left|\omega_{0}\right|<\frac{u_{\max }}{(1+|\beta|)^{2}},
$$

Choosing the control

$$
\nu_{k}(t)=\omega_{0}\left(s_{k}+K\left\langle P_{k} \boldsymbol{c}, e^{i \gamma_{k}}\right\rangle\right), K>0, \omega_{0} \neq 0
$$

forces convergence of all particles to the set of circular formations with radius $\left|\omega_{0}\right|^{-1}$ and direction determined by the sign of $\omega_{0}$.

Proof. Consider the Lyapunov function

$$
V=\frac{1}{2}\langle\boldsymbol{c}, P \boldsymbol{c}\rangle .
$$

The time derivative of $V$ along solutions of (44) is

$$
\dot{V}=\sum_{k=1}^{N}\left\langle P_{k} \boldsymbol{c}, e^{i \gamma_{k}}\right\rangle\left(s_{k}(t)-\omega_{0}^{-1} \nu_{k}\right) .
$$


Using control (53), observe that

$$
\left\langle e^{i \gamma_{k}}, P_{k} \boldsymbol{c}\right\rangle=\frac{\nu_{k}-s_{k}(t) \omega_{0}}{K \omega_{0}}
$$

which implies

$$
\dot{V}=-\sum_{k=1}^{N} \frac{\left(s_{k}(t)-\omega_{0}^{-1} \nu_{k}\right)^{2}}{K}=-\frac{1}{K \omega_{0}^{2}} \sum_{k=1}^{N} \frac{\left(\omega_{0} s_{k}(t)-\nu_{k}\right)^{2}}{K} \leq 0 \forall \nu_{k} .
$$

When $-\nu_{\max } \leq \nu_{k} \leq \nu_{\max }, \dot{V} \leq 0$; otherwise, $\dot{V}$ is strictly less than zero. Applying the invariance principle, solutions of (4) converge to the largest invariant set for which

$$
\left\langle P_{k} \boldsymbol{c}, e^{i \gamma_{k}}\right\rangle=0 .
$$

In this set, (53) evaluates to $\nu_{k}=s_{k}(t) \omega_{0}$ and $\dot{c_{k}}=0$, which implies that the particles travels in a circle with a fixed center. $P_{k} \boldsymbol{c}$ is constant and must evaluate to zero. Because $P$ is spanned by $\mathbf{1}$, this condition is only satisfied when $P \boldsymbol{c}=0$, which implies $c_{k}=c_{j} \forall k, j$. The formation radius (52) satisfies (50) in Lemma 6 . ensuring that the control will drive all particles to a set of asymptotically stable circular formations with radius $\left|\omega_{0}\right|^{-1}$.

By introducing a virtual particle, as was done in Theorem 2, one can similarly establish stabilization of a circular formation with a bounded turn rate at a prescribed center point.

Corollary 1. Consider model (44) where $f_{k}=\beta \in \mathbb{R}, u_{\max }>0$, and $\nu_{\max }=\nu_{k}\left(u_{\max }\right)$. If $\omega_{0}$ satisfies

$$
\left|\omega_{0}\right|<\frac{u_{\max }}{(1+|\beta|)^{2}}
$$

Choosing the control

$$
\nu_{k}(t)=\omega_{0}\left(s_{k}+K\left(\left\langle e^{i \gamma_{k}}, P_{k} \boldsymbol{c}\right\rangle+a_{k 0}\left\langle e^{i \gamma_{k}}, c_{k}-c_{0}\right\rangle\right)\right), K>0, \omega_{0} \neq 0,
$$

where $a_{k 0}=1$ for at least one $k \in 1, \ldots, N$ and zero otherwise, forces convergence to the set of circular formations centered on $c_{0}$ with radius $\left|\omega_{0}\right|^{-1}$ and direction determined by the sign of $\omega_{0}$.

Figure 4 compares a system of particles that conform to the $u_{\max }$ constraint $\sqrt[50]{ }$ with one that does not. By violating (50), the particles do not have the turn-rate control necessary to maintain a circular formation of the chosen radius. This is illustrated in Figure 4(a), using a bound of $u_{\max }=0.1$ and a circular radius of $\left|\omega_{0}\right|^{-1}=10$. The solution does not converge to a circular formation. Figure 4(b) shows convergence to a circular formation with radius $\left|\omega_{0}\right|^{-1}=30.6$, the smallest permissible radius for $u_{\max }=0.1$, according to Corollary 1 .

\section{IV.B. Turn-Rate Constraint in a Rotating Flowfield}

This section shows that the previous results hold for a spatially invariant, rotating flow, $f(t)=\eta_{0} e^{i \Omega t}$.

Lemma 7. Consider circular motion of a particle with radius $\left|\omega_{0}\right|^{-1}$ and flowfield $f(t)=\eta_{0} e^{i \Omega t}$. The maximum turn rate of the particle required to maintain a circular formation is

$$
u_{\text {max }} \triangleq \max _{\gamma_{k}} u\left(\gamma_{k}\right)= \begin{cases}\left|\omega_{0}\right|\left(1+\left|\eta_{0}\right|\right)^{2}-\left|\eta_{0}\right||\Omega| & \text { if } 2\left|\omega_{0}\right|>|\Omega| ; \\ \left|\omega_{0}\right|\left(1-\left|\eta_{0}\right|\right)^{2}+\left|\eta_{0}\right||\Omega| & \text { if } 2\left|\omega_{0}\right| \leq|\Omega| .\end{cases}
$$

The maximum steering control is

$$
\nu_{\text {max }} \triangleq \nu\left(u_{\max }\right)= \begin{cases}\left|\omega_{0}\right|\left(1+\left|\eta_{0}\right|\right) & \text { if } 2\left|\omega_{0}\right|>|\Omega| \\ \left|\omega_{0}\right|\left(1-\left|\eta_{0}\right|\right) & \text { otherwise }\end{cases}
$$

Proof. The turn-rate control $u_{k}$ for a particle with dynamics (1) in a rotating flowfield, $f(t)=\eta_{0} e^{i \Omega t}$, is

$$
u_{k}=u\left(\gamma_{k}^{\prime}\right)=\frac{s_{k}(t) \nu_{k}-\Omega \eta_{0} \cos \left(\gamma_{k}^{\prime}\right)}{s_{k}(t)-\eta_{0} \cos \left(\gamma_{k}^{\prime}\right)}
$$




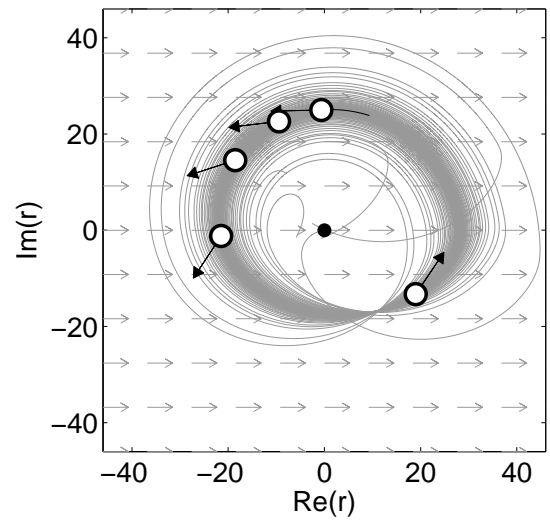

(a) (a)

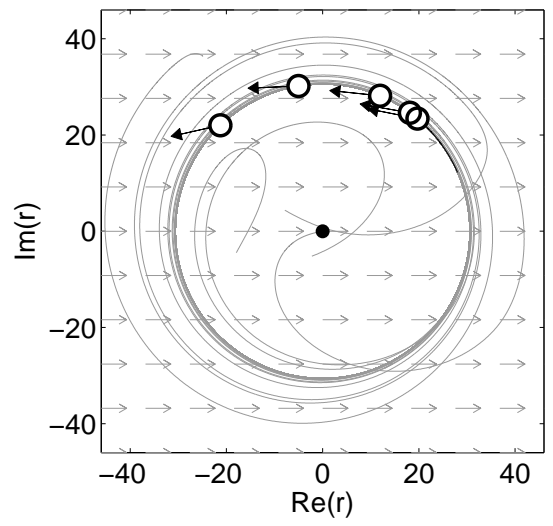

(b) (b)

Figure 4. Effects of a bounded turning control, $u_{\max }=0.35$, in a spatially uniform flowfield with $\beta=0.75$. (a) Circular formation is unobtainable with radius $\left|\omega_{0}\right|^{-1}=10$. (b) Stable circular formation with radius $\left|\omega_{0}\right|^{-1}=30.6$.

where $\gamma_{k}^{\prime}=\gamma_{k}-\Omega t$. The inertial speed $s_{k}(t)$, is ${ }^{22}$

$$
s_{k}(t)=s\left(\gamma_{k}^{\prime}\right)=\eta_{0} \cos \left(\gamma_{k}^{\prime}\right)+\left(1-\eta_{0}^{2} \sin \left(\gamma_{k}^{\prime}\right)^{2}\right)^{\frac{1}{2}} .
$$

Substituting the circular control $\nu_{k}=\omega_{0} s_{k}(t)$ into 63$)$ and finding the extrema with respect to $\gamma_{k}^{\prime}$ yields critical points at $\gamma_{k}^{\prime}=0$ and $\gamma_{k}^{\prime}=\pi$, which implies

$$
u_{k}(0)=\omega_{0}\left(1+\eta_{0}\right)^{2}-\eta_{0} \Omega
$$

and

$$
u_{k}(\pi)=\omega_{0}\left(1-\eta_{0}\right)^{2}+\eta_{0} \Omega
$$

Either (65) or (66) is a maximum point depending on the values of $\eta_{0}, \Omega$ and $\omega_{0}$. If $\eta_{0}>0$ then $u_{k}(0)>u_{k}(\pi)$ when $2\left|\omega_{0}\right|>|\Omega|$. If $\eta_{0}<0$ then $u_{k}(0)>u_{k}(\pi)$ when $2\left|\omega_{0}\right|<|\Omega|$ leading to (61). Under these conditions, (64) becomes $s_{k}(t)=1 \pm|\eta|$ and substituting $s_{k}(t)$ and (61) into 63) gives (62).

Theorem 5. Consider model (44) with $f(t)=\eta_{0} e^{i \Omega t},\left|\eta_{0}\right|<1 \forall t$, and $u_{\max }>0$. If $\omega_{0}$ satisfies

$$
\begin{array}{ll}
\left|\omega_{0}\right|<\left(u_{\max }+\left|\eta_{0}\right||\Omega|\right) /\left(1+\left|\eta_{0}\right|\right)^{2} & \text { if } 2\left|\omega_{0}\right|>|\Omega| \\
\left|\omega_{0}\right|<\left(u_{\max }-\left|\eta_{0}\right||\Omega|\right) /\left(1-\left|\eta_{0}\right|\right)^{2} & \text { if } 2\left|\omega_{0}\right| \leq|\Omega| .
\end{array}
$$

then the control 553) with $\nu_{\max }$ given by (62) forces uniform convergence of solutions to model (44) to the set of circular formations with radius $\left|\omega_{0}\right|^{-1}$ and direction determined by the sign of $\omega_{0}$.

The proof of Theorem 5 follows the proof of Theorem 4 . Since $f(t)$ is time-varying, uniform asymptotic stability is established by the invariance-like principle as in Theorem 3 . The following theorem shows that stabilization of a time-splay formation is possible even with a bounded turn rate, thereby extending $[22$, Theorem 3].

Theorem 6. Consider model (44) with the spatially invariant, rotating flowfield $f=\eta_{0} e^{i \Omega t}$ and $\left|\eta_{0}\right|<1 \forall t$. Also, let $U(\psi)$ be a smooth, rotationally symmetric phase potential. With $\omega_{0}$ such that it satisfies both the

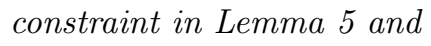

$$
\begin{array}{lll}
\left|\omega_{0}\right|<\left(u_{\max }+\left|\eta_{0}\right||\Omega|\right) /\left(1+\left|\eta_{0}\right|\right)^{2} & \text { if } & 2\left|\omega_{0}\right|>|\Omega| \\
\left|\omega_{0}\right|<\left(u_{\max }-\left|\eta_{0}\right||\Omega|\right) /\left(1-\left|\eta_{0}\right|\right)^{2} & \text { if } & 2\left|\omega_{0}\right| \leq|\Omega| .
\end{array}
$$

Choosing the control

$$
\nu_{k}=\omega_{0} s_{k}\left(1+K\left(s_{k}\left\langle e^{i \gamma_{k}}, P_{k} \boldsymbol{c}\right\rangle-\frac{\partial U}{\partial \psi_{k}} \frac{\omega_{0} s_{k}}{\omega_{0} s_{k}-\Omega}\right)\right), K>0,
$$

and $\nu_{\max }$ given by 62) forces uniform convergence of solutions to the model (44) to the set of circular formations with radius $\left|\omega_{0}\right|^{-1}$ and direction determined by the sign of $\omega_{0}$. 


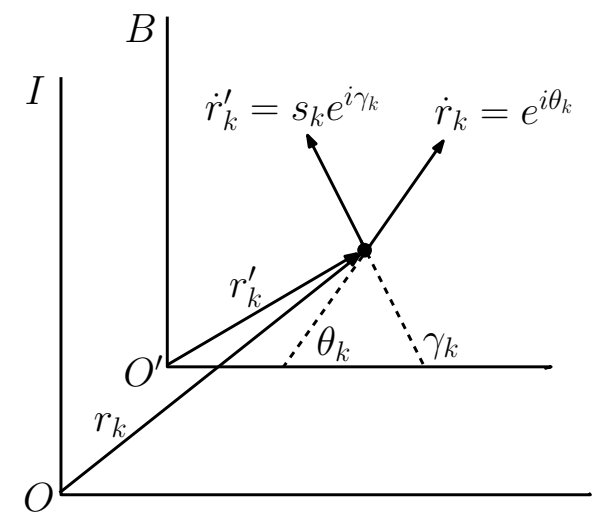

Figure 5. Relationship between inertial frame $\mathcal{I}$ and accelerating frame $\mathcal{B}$ with two path trajectories.

Proof. Using the control $(69)$, observe that

$$
\frac{\nu_{k}-\omega_{0} s_{k}(t)}{K \omega_{0} s_{k}(t)}=s_{k}(t)\left\langle e^{i \gamma_{k}}, P_{k} \boldsymbol{c}\right\rangle-\frac{\partial U}{\partial \psi_{k}} \frac{\omega_{0} s_{k}(t)}{\omega_{0} s_{k}(t)-\Omega} .
$$

Consider the Lyapunov function

$$
V(\boldsymbol{r}, \boldsymbol{\gamma})=\frac{1}{2}\langle\boldsymbol{c}, P \boldsymbol{c}\rangle+\frac{T}{2 \pi} U(\boldsymbol{\psi}) .
$$

Taking time-derivative of (71) yields

$$
\begin{aligned}
\dot{V} & =\sum_{k=1}^{N}\left\langle e^{i \gamma_{k}}, P_{k} \boldsymbol{c}\right\rangle\left(s_{k}-\omega_{0}^{-1} \nu_{k}\right)+\frac{T}{2 \pi} \frac{\partial U}{\partial \psi_{k}} \dot{\psi}_{k} \\
& =\sum_{k=1}^{N}\left(s_{k}\left\langle e^{i \gamma_{k}}, P_{k} \boldsymbol{c}\right\rangle-\frac{\partial U}{\partial \psi_{k}} \frac{\omega_{0} s_{k}}{\omega_{0} s_{k}-\Omega}\right)\left(\frac{\omega_{0} s_{k}-\nu_{k}}{\omega_{0} s_{k}}\right) .
\end{aligned}
$$

We substitute 70 into 72 to obtain

$$
\dot{V}=\frac{1}{K \omega_{0}^{2}} \sum_{k=1}^{N} \frac{\left(\nu_{k}-\omega_{0} s_{k}(t)\right)^{2}}{\left(s_{k}(t)\right)^{2}} \leq 0 \forall \nu_{k} .
$$

$\dot{V}$ is strictly less than zero except when $-\nu_{\max } \leq \nu_{k} \leq \nu_{\max }$. In this interval $\dot{V} \leq 0$. This case is covered by Theorem 3 , for which it is less than or equal to zero. The rest of the proof follows the proof of Theorem 3 .

\section{Coordinated Encirclement of a Maneuvering Target}

In this section consider the scenario of multiple unmanned vehicles following a maneuvering target so that the target forms the center of a circular formation. A non-rotating reference frame $\mathcal{B}$ is defined whose origin $O^{\prime}$ is fixed to the target. $O^{\prime}$ moves with respect to the inertial frame $\mathcal{I}$ with a velocity and acceleration equal to the target velocity. Two path frames are utilized for particle $k$ as shown in Figure 5 . With no external flowfield, the kinematics in the inertial frame are given by model (3) with $f_{k}(t)=0$. Integrating the following equations of motion (see Appendix VI) yields $r_{k}^{\prime}$ :

$$
\begin{aligned}
\dot{r}_{k}^{\prime} & =s_{k}(t) e^{i \gamma_{k}} \\
\dot{\gamma}_{k} & =\nu_{k},
\end{aligned}
$$

where $\dot{s}_{k}(t)$ given by 90 . The inertial trajectory is

$$
r_{k}(t)=r_{k}^{\prime}(t)+\frac{1}{2}\left(a_{x}+i a_{y}\right) t^{2}+\left(v_{x}(0)+i v_{y}(0)\right) t .
$$




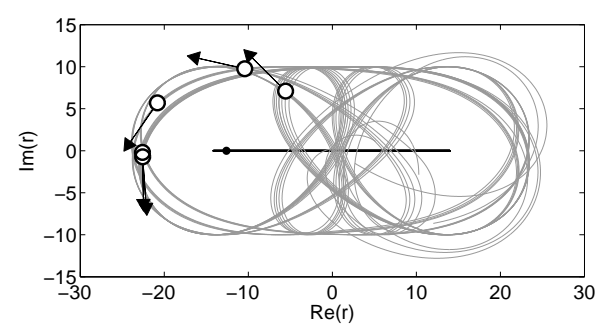

(a) Inertial frame

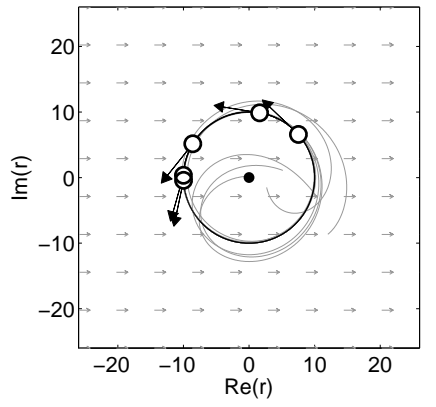

(b) Target frame, $t=400 \mathrm{~s}$

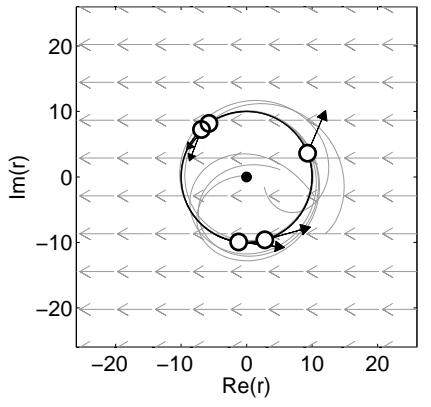

(c) Target frame, $t=600 \mathrm{~s}$

Figure 6. Encirclement of a maneuvering target that is accelerating back and forth along a single trackline.

The following examples utilize model (4) with (90) and assign the center point of the circular formation to a target in frame $\mathcal{B}$. The target-encirclement scenario is illustrated with three examples. In Section V.A a target is considered that is continuously accelerating and decelerating without turning. The magnitude of the velocity is always changing. Section V.B illustrates the coordinated encirclement of a target that is traversing a circle of fixed radius and thus constantly changing its velocity direction while maintaining a fixed magnitude. For this flowfield the time-phase parameter is used to regulate the temporal spacing of the particles. Section V.C depicts the velocity of an actual target driving through an urban environment. This example shows the utility of the algorithms developed in Section III to encircle a target in an estimated flowfield. Additionally, a turning-rate constraint is imposed on the vehicles as discussed in Section IV] All of the examples illustrate realistic maneuvers of a mobile ground vehicle. In all the target-tracking examples each autonomous vehicle measures the relative distance between it and the target.

\section{V.A. Coordinated Encirclement of a Variable-Speed Target}

This example replicates a target that is accelerating back and forth along a single trackline. Since the target velocity only varies in magnitude, it can be aligned with the real axis of an inertial frame without loss of generality. This scenario is illustrated using control 60 and target acceleration

$$
a_{x}(t)= \begin{cases}\frac{-4 l}{T}, & \bmod (t, T)<\frac{T}{2} \\ \frac{4 l}{T}, & \bmod (t, T) \geq \frac{T}{2},\end{cases}
$$

where $T$ and $l$ represent the period and the maximum amplitude of the velocity, respectively. The target speed is greatest in the middle portion of the track and slows down and reverses direction at the edges.

Figure 6 illustrates the encirclement result of a target traversing with a period $T=150$ seconds and a maximum velocity equal to $75 \%$ of the particle's velocity $(l=0.75)$. Figure $6(\mathrm{a})$ shows the simulated results in an inertial reference frame. The target is aligned with the real axis and travels left and right without turning. In this figure it is not apparent that the particles converge to a circular formation. However, this is readily seen in the target-centered frame as illustrated with Figures 6(b) and 6(c). The target-centered figures illustrate the velocity by using the equivalent spatially uniform flowfield at that instance in time. Figure 6(b) shows the formation at $t=400$ seconds; figure 6(c) shows the formation at $t=600$ seconds, when the target is traveling at its maximum velocity of $l=0.75$.

\section{V.B. Coordinated Encirclement of a Turning Target}

This example illustrates the particle behavior as they follow a target performing a constant-rate turn of radius $\rho$. The target acceleration is $a_{O}(t)=\left(\eta_{0}^{2} / \rho\right) i e^{i 2 \pi t / T}$. The target has a fixed speed $\eta_{0}$ and a constantly changing direction of motion. Figure 7 illustrates the results of using control algorithm (60) with speed $\eta_{0}=0.5$ ( $50 \%$ of the particle's velocity) and radius of curvature $\rho=39.79$. The period of time it takes the target to traverse the circle is $T=500$ seconds. Figure $7(\mathrm{a})$ illustrates the results in the inertial frame, showing the target track as it travels in a circle and the resulting particle tracks. Figures 7(b) and 7(c) 


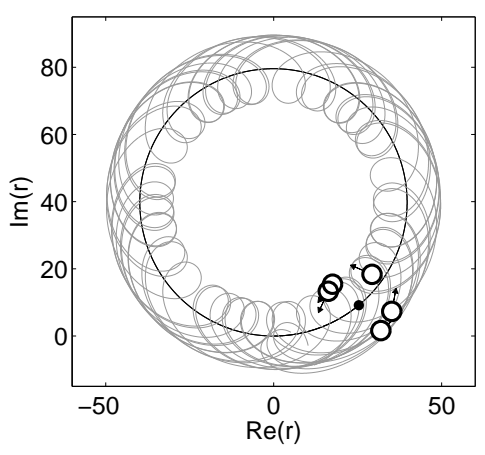

(a) Inertial frame

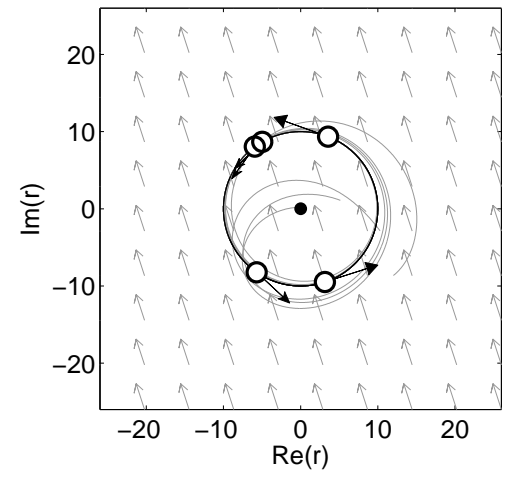

(b) Target frame, $t=400 \mathrm{~s}$

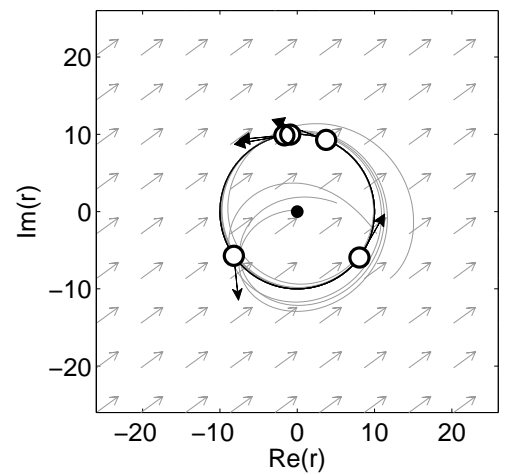

(c) Target frame, $t=800 \mathrm{~s}$

Figure 7. Encirclement of a maneuvering target that is turning at a constant rate.

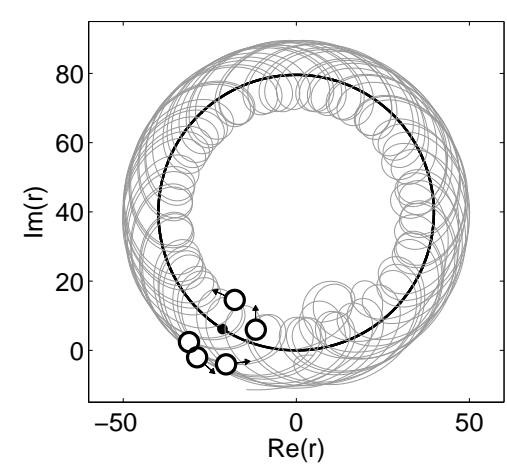

(a) Inertial frame

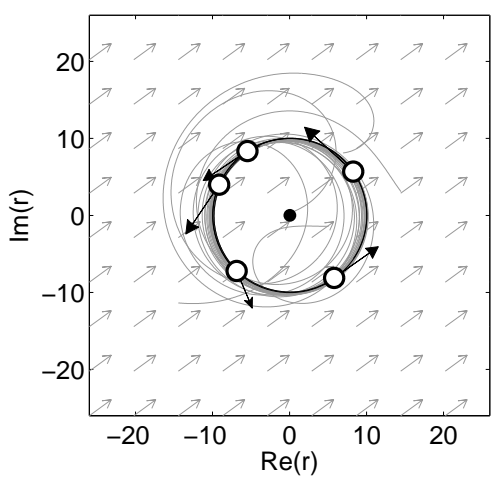

(b) Target frame, $t=800 \mathrm{~s}$

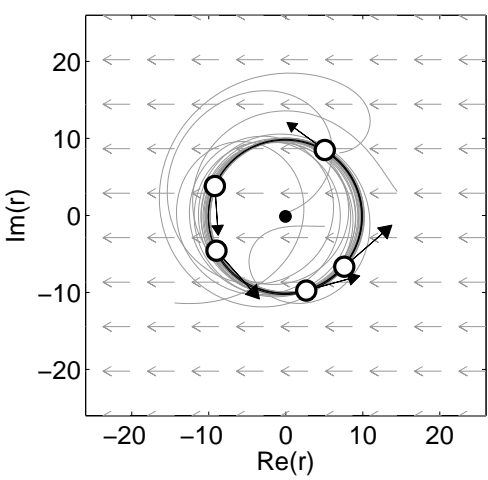

(c) Target frame, $t=1000 \mathrm{~s}$

Figure 8. Coordinated encirclement of a maneuvering target that is turning at a constant rate.

display the simulation results in a target-fixed frame at $t=400$ and $t=800$ seconds, respectively. In these figures the particles quickly converge to a circular formation and maintain that formation even as the target accelerates.

The turning-target scenario is also used to illustrate control law $(69)$, which drives the particles to a time-splay formation. Figure 8 illustrates this example with $N=5$ particles centered on a target circling at an angular rate of $\Omega=2 \pi / T$, period $T=500 s$, and target speed, $\eta_{0}=0.5$. Setting $\omega_{0}=0.1$ satisfies the requirement in Lemma 5. Figure 8(a) depicts the target and particles in the inertial frame. Figures 8(b) and $8(\mathrm{c})$ show the convergence to the time-splay configuration in the target-centered reference frame at $t=800$ and $t=1000$ seconds. These figures show that the particles converge to a time-splay formation even as the target accelerates.

\section{V.C. Coordinated Encirclement of Maneuvering Target in an Urban Environment}

In this example the work of Sections [II] and IV] are combined to encircle a maneuvering target moving with an unknown velocity. Control algorithm 25) was used to estimate the velocity of the target. Additionally, a bounded turn-rate constraint is placed on the autonomous vehicles. The combined particle model for this simulation is

$$
\begin{aligned}
& \dot{\hat{r}}_{k}=\hat{s}_{k}(t) e^{i \hat{\gamma}_{k}} \\
& \dot{\hat{\gamma}}_{k}=\operatorname{sat}\left(\nu_{k} ; \nu_{\max }\right) .
\end{aligned}
$$

A set of GPS waypoints is used to model realistic behavior of a vehicle traveling in an urban environment. The vehicle tracks were collected during a twenty-minute interval while driving through a Washington, D.C. 


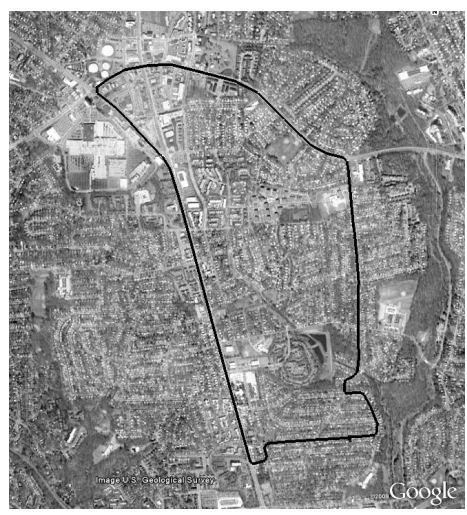

(a) Target route

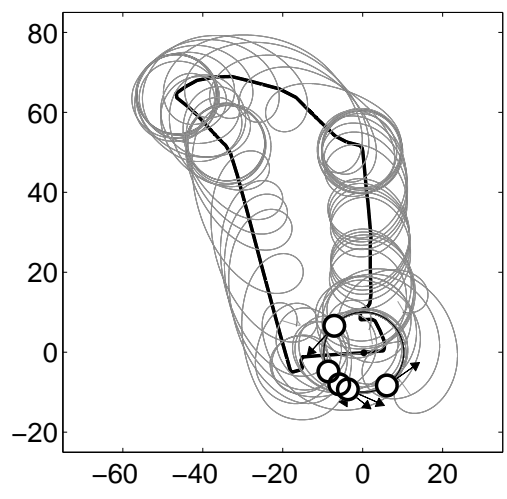

(b) Inertial frame

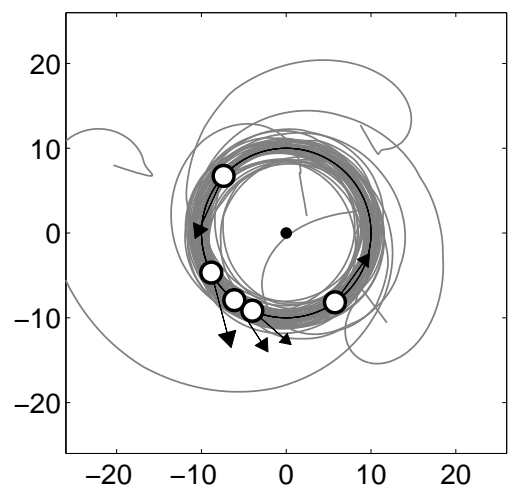

(c) Target frame, $t=1350 \mathrm{~s}$

Figure 9. Coordinated encirclement of a target moving through urban traffic.

suburb. The track included stops, turns, and other kinematic behavior typical in urban traffic. The maximum velocity of the target was $18.23 \mathrm{~m} / \mathrm{s}$ (approx. $40 \mathrm{miles} / \mathrm{hr}$ ). It is assumed that the optimal velocity of the autonomous vehicles is $35 \mathrm{~m} / \mathrm{s}$. The velocity of the target and autonomous vehicles are normalized such that the autonomous vehicles travel at unit speed. (The normalized target velocity is $52 \%$ of the platform speed.) A turn-rate of $u_{\max }=0.23 \mathrm{rad} / \mathrm{s}$ is imposed on the vehicles, which corresponds to a UAV maximum bank angle of $40^{\circ} .{ }^{10}$ Following 50 from Lemma 6 a formation radius of $\left|\omega_{0}\right|^{-1}=10$ was choosen.

Figure 9 shows the results from the urban-traffic scenario. Figure 9(a) displays the GPS waypoints on a street map of the area. The movement of the target-fixed frame was generated from these waypoints by converting the GPS latitude and longitude points into a topocentric North-East-Down (NED) coordinate frame centered on the starting location. In this frame, the distances between the waypoints and their associated timestamps were used to determine the velocity of the target vehicle. The target velocity is piecewise constant because it is constructed out of a discrete set of waypoints and does not consider acceleration. Discontinuities of the velocity are especially pronounced when the vehicle turns sharply as both the direction and magnitude may change suddenly. Although the velocity of the target is being estimated, the vehicles are able to measure the relative position between itself and the target. Figure 9(b) displays both the target and vehicle tracks in the inertial frame. (The distance units are based upon the normalized speed of the ground vehicle.) The figure shows separations in the vehicle tracks that correspond to the velocity of the target. When the target is traveling quickly the cyclic tracks are spaced farther apart than in the slower moving parts of the track. Points at which the target is stopped (due to traffic lights) are evident by the track circle over that point. Figure 9(c) shows the circular formation in the target-fixed frame for the twenty minute time interval. This figure highlights the effect of the velocity discontinuities on the formation: particles exhibit a period of transient behavior after each maneuver, before converging to a circular formation.

\section{Conclusion}

Cooperative control improves the capability of autonomous vehicles to gather information, track targets and perform other mission objectives. In this paper, each autonmous vehicle is represented by a Newtonian-particle that travels at unit speed and is subject to a steering control applied perpendicularly to the particle's velocity relative to the flow. We present decentralized control algorithms that regulate the formation of autonomous vehicles while operating in unknown, spatially invariant flowfields of moderate strength. Additionally circular and time-splay configurations are derived for unknown rotating flowfields. These algorithms are shown to be robust to bounds on the turn rate of the vehicle. Application simulations are presented which illustrate the capability to cooperatively encircle maneuvering targets that turn, accelerate and operate in an urban environment. Future work seeks to expand the class of flowfields for which these results apply. 


\section{Appendix A}

In this appendix the real portion of the eigenvalues of matrix $B$ defined in $(28)$ is derived.

$$
\begin{aligned}
\lambda & =\frac{-\left(K_{1}-\Omega i\right) \pm \sqrt{\left(K_{1}-\Omega i\right)^{2}-4\left(-K_{1} \Omega i+K_{2}\right)}}{2} \\
& =\frac{-K_{1}+\Omega i \pm \sqrt{\left(K_{1}^{2}-\Omega^{2}-4 K_{2}\right)+\left(2 K_{1} \Omega\right) i}}{2} .
\end{aligned}
$$

The real part of the eigenvalues are

$$
\operatorname{Re}(\lambda)=\frac{-K_{1} \pm \operatorname{Re}\left(\sqrt{\left(K_{1}^{2}-\Omega^{2}-4 K_{2}\right)+\left(2 K_{1} \Omega\right) i}\right)}{2} .
$$

To find the real part of the square root term, which contains a complex number, observe that

$$
\operatorname{Re}(\sqrt{a+b i})=\operatorname{Re}\left(\sqrt{\rho e^{\phi i}}\right)=\sqrt{\rho} \cos \frac{\phi}{2}
$$

with $\rho=\sqrt{a^{2}+b^{2}}$ and $\cos \phi=a / \rho$. Using the half-angle formula for $\cos \phi=\sqrt{(1+\cos 2 \phi) / 2}$, the real part of square root becomes

$$
\operatorname{Re}(\sqrt{a+b i})=\sqrt{\rho} \sqrt{\left(\frac{1+\frac{a}{\rho}}{2}\right)}=\sqrt{\frac{\rho+a}{2}} .
$$

Substituting into 76 yields

$$
\operatorname{Re}(\lambda)=\frac{-K_{1}}{2} \pm \frac{\sqrt{\sqrt{\left(K_{1}^{2}-\Omega^{2}-4 K_{2}\right)^{2}+4 K_{1}^{2} \Omega^{2}}+\left(K_{1}^{2}-\Omega^{2}-4 K_{2}\right)}}{2 \sqrt{2}},
$$

which is the real portion of the eigenvalues of matrix $B$.

\section{Appendix B}

In this appendix we develop the inertial speed $s_{k}(t)$ for a Newtonian particle traveling in an accelerating frame. With no external flowfield, the kinematics in the inertial frame are

$$
\begin{aligned}
& \dot{r}_{k}=e^{i \theta_{k}} \\
& \ddot{r}_{k}=\dot{\theta}_{k} i e^{i \theta_{k}} .
\end{aligned}
$$

The steering control is $\dot{\theta}_{k}=u_{k}$. Let $r_{k}^{\prime}$ be the position of the particle $k$ relative to $O^{\prime}$. The inertial kinematics relative to $O^{\prime}$ are

$$
\begin{aligned}
& \dot{r}_{k}^{\prime} \triangleq s_{k}(t) e^{i \gamma_{k}} \\
& \ddot{r}_{k}^{\prime}=\dot{s}_{k}(t) e^{i \gamma_{k}}+s_{k}(t) \dot{\gamma}_{k} i e^{i \gamma_{k}} .
\end{aligned}
$$

$s_{k}(t)$ and $\gamma_{k}$ are the magnitude and orientation of $\dot{r}_{k}^{\prime}$. Let $r_{O^{\prime}}$ be the position of $O^{\prime}$ with respect to $O$. By vector addition we have

$$
\ddot{r}_{k}^{\prime}=\ddot{r}_{k}-\ddot{r}_{O^{\prime}} .
$$

The velocity and acceleration between frames $\mathcal{I}$ and $\mathcal{B}$ is defined to be $\dot{r}_{O}^{\prime}=v_{O}=v_{x}+i v_{y}$ and $\ddot{r}_{O}^{\prime}=$ $a_{O}=a_{x}+i a_{y}$, respectively. To find the equations of motion for the particle $k$ in frame $\mathcal{B}$, substitute (78) and 80 into 81 , which yields

$$
\dot{s}_{k}(t) e^{i \gamma_{k}}+s_{k}(t) \dot{\gamma}_{k} i e^{i \gamma_{k}}=u_{k} i e^{i \theta_{k}}-a_{x}-i a_{y} .
$$

From Figure 5 and Euler's formula, observe that

$$
\begin{aligned}
i e^{i \theta_{k}} & =\sin \left(\gamma_{k}-\theta_{k}\right) e^{i \gamma_{k}}+\cos \left(\gamma_{k}-\theta_{k}\right) i e^{i \gamma_{k}} \\
a_{x} & =a_{x}\left(\cos \gamma_{k} e^{i \gamma_{k}}-\sin \gamma_{k} i e^{i \gamma_{k}}\right) \\
a_{y} & =a_{y}\left(\sin \gamma_{k} e^{i \gamma_{k}}+\cos \gamma_{k} i e^{i \gamma_{k}}\right) .
\end{aligned}
$$


Substituting (83)-85] into 82 yields

$$
\begin{aligned}
\dot{s}_{k}(t) & =u_{k} \sin \left(\gamma_{k}-\theta_{k}\right)-a_{x} \cos \gamma_{k}-a_{y} \sin \gamma_{k} \\
\dot{\gamma}_{k} & =\frac{1}{s_{k}(t)}\left(u_{k} \cos \left(\gamma_{k}-\theta_{k}\right)+a_{x} \sin \gamma_{k}-a_{y} \cos \gamma_{k}\right) \triangleq \nu_{k} .
\end{aligned}
$$

Solving (87) for $u_{k}$ and substituting into 867) gives

$$
\dot{s}_{k}(t)=\left(s_{k}(t) \nu_{k}-a_{x} \sin \gamma_{k}+a_{y} \cos \gamma_{k}\right) \tan \left(\gamma_{k}-\theta_{k}\right)-a_{x} \cos \gamma_{k}-a_{y} \sin \gamma_{k} .
$$

To eliminate $\theta_{k}$ from 88 use

$$
\begin{aligned}
s_{k}(t) e^{i \gamma_{k}} & =e^{i \theta_{k}}-v \\
s_{k}(t) e^{i\left(\gamma_{k}-\theta_{k}\right)} & =1-v e^{-i \theta_{k}} .
\end{aligned}
$$

Equating the real and imaginary parts of the above equations yields

$$
\begin{aligned}
& s_{k}(t) \cos \gamma_{k}=\cos \theta_{k}-v_{x} \\
& s_{k}(t) \sin \gamma_{k}=\sin \theta_{k}-v_{y}
\end{aligned}
$$

and

$$
\begin{aligned}
s_{k}(t) \cos \left(\gamma_{k}-\theta_{k}\right) & =1-v_{x} \cos \theta_{k}-v_{y} \sin \theta_{k} \\
& =1-v_{x}\left(v_{x}+s_{k}(t) \cos \gamma_{k}\right)-v_{y}\left(v_{y}+s_{k}(t) \sin \gamma_{k}\right) \\
s_{k}(t) \sin \left(\gamma_{k}-\theta_{k}\right) & =v_{x} \sin \theta_{k}-v_{y} \cos \theta_{k} \\
& =v_{x} s_{k}(t) \sin \gamma_{k}-v_{y} s_{k}(t) \cos \gamma_{k}
\end{aligned}
$$

which gives

$$
\tan \left(\gamma_{k}-\theta_{k}\right)=\frac{v_{x} s_{k}(t) \sin \gamma_{k}-v_{y} s_{k}(t) \cos \gamma_{k}}{\left(1-v_{x}^{2}-v_{y}^{2}-v_{x} s_{k}(t) \cos \gamma_{k}-v_{y} s_{k}(t) \sin \gamma_{k}\right)}
$$

Substituting 890 into 88 yields

$$
\begin{aligned}
\dot{s}_{k}(t)= & \frac{\left(s_{k}(t) \nu_{k}-a_{x} \sin \gamma_{k}+a_{y} \cos \gamma_{k}\right)\left(v_{x} s_{k}(t) \sin \gamma_{k}-v_{y} s_{k}(t) \cos \gamma_{k}\right)}{1-v_{x}^{2}-v_{y}^{2}-v_{x} s_{k}(t) \cos \gamma_{k}-v_{y} s_{k}(t) \sin \gamma_{k}} \\
& -a_{x} \cos \gamma_{k}-a_{y} \sin \gamma_{k}
\end{aligned}
$$

\section{Acknowledgments}

This material is based upon work supported by the National Science Foundation under Grant No. CMMI0928416 and the Office of Naval Research Grant No. N00014-09-1-1058.

\section{References}

${ }^{1}$ Justh, E. W. and Krishnaprasad, P. S., "Equilibria and Steering Laws for Planar Formations," Systems and Control Letters, Vol. 52, No. 1, 2004, pp. 25-38.

${ }^{2}$ Sepulchre, R., Paley, D. A., and Leonard, N. E., "Stabilization of planar collective motion: All-to-all communication," IEEE Trans. Automatic Control, Vol. 52, No. 5, 2007, pp. 811-824.

${ }^{3}$ Sepulchre, R., Paley, D. A., and Leonard, N. E., "Stabilization of planar collective motion with limited communication," IEEE Trans. Automatic Control, Vol. 53, No. 3, 2008, pp. 706-719.

${ }^{4}$ Elston, J. and Frew, E., "Unmanned Aircraft Guidance for Penetration of Pre-Tornadic Storms," Proc. AIAA Guidance, Navigation and Control Conf. and Exhibit (electronic), No. AIAA-2008-6513, Honolulu, Hawaii, 2008, (16 pages).

${ }^{5}$ Frew, E. W., Lawrence, D. A., and Morris, S., "Coordinated Standoff Tracking of Moving Targets Using Lyapunov Guidance Vector Fields," J. Guidance, Control, and Dynamics, Vol. 31, No. 2, 2008, pp. 290-306.

${ }^{6}$ Summers, T. H., Akella, M. R., and Mears, M. J., "Coordinated Standoff Tracking of Moving Targets: Control Laws and Information Architectures," J. Guidance, Control, and Dynamics, Vol. 32, No. 1, 2009, pp. 56-69.

${ }^{7}$ Olfati-Saber, R. and Murray, R., "Graph Rigidity and Distributed Formation Stabilization of Multi-Vehicle Systems," IEEE Conference on Decision and Control, Vol. 3, 2002, pp. 2965-2971. 
${ }^{8}$ Kingston, D. B., Decentralized Control of Multiple UAVs for Perimeter and Target Surveillance, Ph.D. thesis, Department of Electrical and Computer Engineering, Brigham Young University, Provo, Utah, December 2007.

${ }^{9}$ Fax, J. A. and Murray, R. M., "Information Flow and Cooperative Control of Vehicle Formations," IEEE Trans. Automatic Control, Vol. 49, No. 9, 2004, pp. 1465-1476.

${ }^{10}$ Wise, R. and Rysdyk, R., "UAV Coordination for Autonomous Target Tracking," AIAA Guidance, Navigation, and Control Conference (electronic), No. AIAA-2006-6453, AIAA, Keystone, Colorado, August 2006, (22 pages).

${ }^{11}$ Techy, L., Woolsey, C. A., and Schmale, D. G., "Path Planning for Efficient UAV Coordination in Aerobiological Sampling Missions," Proc. IEEE Conf. Decision and Control, Cancun, Mexico, December 2008, pp. 2814-2819.

${ }^{12}$ Elston, J. and Frew, E., "Unmanned Aircraft Guidance for Penetration of Pre-Tornadic Storms," J. Guidance, Control, and Dynamics, Vol. 33, No. 1, 2010, pp. 99-107.

${ }^{13}$ Fiorelli, E., Leonard, N., Bhatta, P., Paley, D., Bachmayer, R., and Fratantoni, D., "Multi-AUV control and adaptive sampling in Monterey Bay," IEEE Journal of Oceanic Engineering, Vol. 31, No. 4, 2006, pp. 935.

${ }^{14}$ Hsieh, C., Jin, Z., Marthaler, D., Nguyen, B., Tung, D., Bertozzi, A., and Murray, R., "Experimental validation of an algorithm for cooperative boundary tracking," Proc. of the American Control Conference, Vol. 2, 2005, pp. $1078-1083$.

${ }^{15}$ Klein, D. J. and Morgansen, K. A., "Controlled Collective Motion for Trajectory Tracking," Proc. 2006 American Control Conf., Minneapolis, Minnesota, June 2006, pp. 5269-5275.

${ }^{16}$ Ceccarelli, N., Enright, J. J., Frazzoli, E., Rasmussen, S. J., and Schumacher, C. J., "Micro UAV Path Planning for Reconnaissance in Wind," Proc. 2007 American Control Conf., New York City, New York, July 2007, pp. 5310-5315.

${ }^{17}$ McGee, T. G., Spry, S., and Hedrick, J. K., "Optimal Path Planning in a Constant Wind with a Bounded Turning Rate," Proc. AIAA Conf. Guidance, Navigation, and Control (electronic), No. AIAA 2005-6186, San Francisco, California, August 2005, (11 pages).

${ }^{18}$ Paley, D. A., "Stabilization of Collective Motion in a Uniform and Constant Flow Field" Proc. AIAA Guidance, Navigation and Control Conf. and Exhibit (electronic), No. AIAA-2008-7173, Honolulu, Hawaii, August 2008, (8 pages).

${ }^{19}$ Paley, D. A. and Peterson, C., "Stabilization of Collective Motion in a Time-Invariant Flow Field," J. Guidance, Control, and Dynamics, Vol. 32, No. 3, 2009, pp. 771-779.

${ }^{20}$ Paley, D. A., Techy, L., and Woolsey, C. A., "Coordinated Perimeter Patrol with Minimum-Time Alert Response," AIAA Guidance, Navigation, and Control Conference (electronic), No. AIAA-2009-6210, AIAA, August 2009, (10 pages).

${ }^{21}$ Techy, L., Paley, D. A., and Woolsey, C. A., "UAV Coordination on Time-Optimal Paths in Wind," J. Guidance, Control, and Dynamics (submitted).

${ }^{22}$ Peterson, C. and Paley, D., "Multi-Vehicle Coordination in a Time-Varying Flowfield," Proc. AIAA Guidance, Navigation and Control Conf. and Exhibit (electronic), No. AIAA-2009-6117, Chicago, Illinois, 2009.

${ }^{23}$ Khalil, H. K., "Advanced Stability Analysis," Nonlinear Systems, chap. 8, Prentice Hall, 3rd ed., 2002, pp. 303-338.

${ }^{24}$ Richards, A. and How, J., "Aircraft trajectory planning with collision avoidance using mixed integer linear programming," Proc. of the American Control Conference, Vol. 3, 2002, pp. 1936-1941. 